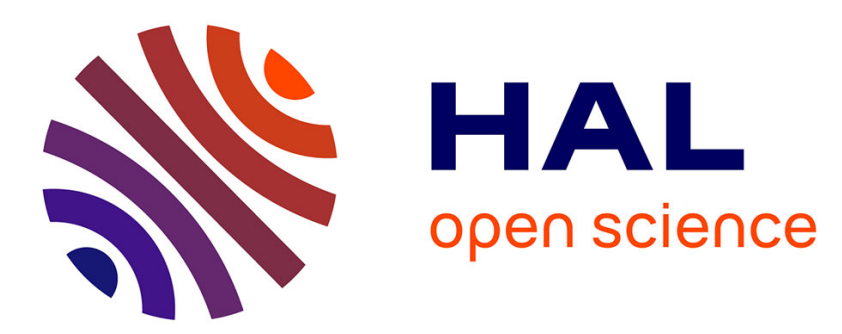

\title{
Mixture model for two-phase flows with high density ratios: A conservative and realizable SPH formulation
}

Thomas Fonty, Martin Ferrand, Agnès Leroy, Antoine Joly, Damien Violeau

\section{To cite this version:}

Thomas Fonty, Martin Ferrand, Agnès Leroy, Antoine Joly, Damien Violeau. Mixture model for twophase flows with high density ratios: A conservative and realizable SPH formulation. International Journal of Multiphase Flow, 2019, 111, pp.158-174. 10.1016/j.ijmultiphaseflow.2018.11.007 . hal03248980

\section{HAL Id: hal-03248980 \\ https://hal.science/hal-03248980}

Submitted on 3 Jun 2021

HAL is a multi-disciplinary open access archive for the deposit and dissemination of scientific research documents, whether they are published or not. The documents may come from teaching and research institutions in France or abroad, or from public or private research centers.
L'archive ouverte pluridisciplinaire HAL, est destinée au dépôt et à la diffusion de documents scientifiques de niveau recherche, publiés ou non, émanant des établissements d'enseignement et de recherche français ou étrangers, des laboratoires publics ou privés. 


\title{
Mixture model for two-phase flows with high density ratios: A conservative and realizable SPH formulation
}

\author{
Thomas Fonty ${ }^{\mathrm{a}, \mathrm{c}, *}$, \\ Martin Ferrand ${ }^{\mathrm{b}}$, Agnès Leroy ${ }^{\mathrm{a}, \mathrm{c}}$, Antoine Joly ${ }^{\mathrm{a}, \mathrm{c}}$, Damien Violeau ${ }^{\mathrm{a}, \mathrm{c}}$ \\ ${ }^{a} L N H E, E D F R \& D$ \\ ${ }^{b} M F E E, E D F R E D$ \\ ${ }^{c}$ Saint-Venant Laboratory for Hydraulics (Ecole des Ponts, EDF R\&D, CEREMA, UPE) \\ 6 quai Watier, 78400 Chatou, France
}

\begin{abstract}
The numerical modelling of two-phase mixture flows with high density ratios (e.g. water/air) is challenging. Multiphase averaged models with volume fraction representation encompass a simple way of simulating such flows: mixture models with relative velocity between phases. Such approaches were implemented in SPH (Smoothed Particle Hydrodynamics) using a mass-weighted definition of the mixture velocity, but with limited validation. Instead, to handle high density ratios, a mixture model with a volumetric mixture velocity is developed in this work. To avoid conservation issues raised by the discretization of the relative material displacement contribution in the volume fraction equation, a formulation on phase volumes is derived following a finite volume reasoning. Conservativity, realizability, limit behaviour for single-phase flow are the leading principles of this derivation. Volume diffusion is added to prevent development of instabilities due to the colocated nature of SPH. This model is adapted to the semi-analytical SPH wall boundary conditions. Running on GPU, this approach is successfully applied to the separation of phases in a settling tank with low to high density ratios. An analytical solution on a two-phase mixture Poiseuille flow is also used to check the accuracy of the numerical implementation. Then, a Rayleigh-Taylor instability test case is performed to compare with multi-fluid SPH. Finally, a comparison with experimental and numerical data is made on a sand dumping case; this highlights some limits of this mixture model. Keywords: Smoothed Particle Hydrodynamics, two-phase mixture flows, large density ratios
\end{abstract}

\footnotetext{
* Corresponding author

Email address: thomas.fonty@edf.fr (Thomas Fonty)
} 


\section{Introduction}

Multi-component flows with high density ratio play a prominent role in many engineering applications and imply complex strong flow dynamics (e.g. air-water turbulent mixing in hydraulic jump or over dam spillways as in Wan et al. (2018)). The SPH (Smoothed Particle Hydrodynamics) method therefore appears as a natural way to deal with such cases, due to its ability to model such highly deformed flows (e.g. dam break or wedge entry in water detailed in Gong et al. (2016)). Multi-fluid SPH models have been extensively studied to take advantage of the good ability of the method in tracking the interface between different phases. Particular attention was paid to high density ratio cases applied to air-water cases such that dam-break or rising-bubble cases in Colagrossi \& Landrini (2003), or Rayleigh-Taylor instability and gravity currents for moderate density ratios in Grenier (2009), Monaghan \& Rafiee (2012). However, accurately modelling multi-fluid phenomena with the usual SPH approach in the air-water case requires choosing a particle discretisation of less than the size of an air bubble or water drop, which leads to prohibitive computational cost at the scale of practical interest for engineering applications.

Local instant formulation for multi-phase flows being generally beyond computational capability, multi-phase averaged models have been developed, as in Ishii \& Hibiki (2011). Due to the averaging process, the interface between phases is no longer explicitly tracked. One gets a continuum in which phases are followed through a volume fraction representation. This kind of model encompasses two main approaches?

- Two-fluid models. Each phase is described by a continuity and a momentum equations. Interfacial interactions introduce additional terms in these equations. They are closed by constitutive laws, depending on the flow regime, but their determination can prove to be tedious;

- Mixture models. The flow is seen as a single-fluid flow with one continuity and one momentum equation that rule the evolution of mixture quantities (mixture density and velocity, combinations of phase properties to be defined) complemented by an additional equation for the mass conservation of one phase. Some additional terms linked to the relative velocity between phases, computed through a closure law depending on the flow regime, appear in

\footnotetext{
${ }^{1}$ In view of the hydraulic applications, energy considerations are neglected in what follows.
} 
these equations. In the absence of relative velocity, one has a homogeneous fluid model.

These models are of particular interest for modelling flows with small-scale interfaces (e.g. dispersed air phase in a water flow) and are usually implemented using the finite volume approach, as in Gallouët et al. (2004). A prominent point consists in solving the volume fraction evolution properly as underlined by Rusche (2002). However, free surface tracking is not an easy task with meshbased approaches, so that these kinds of multi-phase models were also developed in SPH to deal with sediment-laden flows with a two-fluid model in Shi et al. (2017) or separated flows with mixture models in Grenier (2009). It introduces an original way of dealing with phases in SPH: instead of having different sets of particles for each phase, each individual SPH particle is now able to carry the different phases with their respective volume fractions. First studies employing volume fraction representation in SPH were done in an astrophysical framework to study gas/dust mixtures in Monaghan \& Kocharyan (1995) and Monaghan (1997), following a two-fluid model, further applied to sedimentation in (Kwon \& Monaghan, 2015). The unique velocity field for the motion of SPH particles makes the mixture model enticing. It is close to the usual single-phase set of equations and can therefore be implemented with limited effort. It is the approach taken here. The reduced number of equations to solve is expected to limit the computations compared to two-fluid approaches with a different set of particles for each phase, as described in (Shi et al. 2017) or (Kwon \& Monaghan, 2015). Mixture models have already been tested in SPH:

- Price \& Laibe (2015) proposed a mixture model applied to dust settling in protoplanetary discs by rewriting the two-phase equations through the introduction of new variables, combinations of phase variables, to overcome some issues inherent to the two-fluid approach (overdamping at high drag, artificial trapping of dust particles);

- In the graphics community, a mixture model with a volume fraction representation was implemented to capture a wide range of physical phenomena in Ren et al. (2014) but without quantitative validation. This work includes a closure for diffusion velocities (velocity of the phases relative to the mass centre, equal to the difference of the phase velocities and massweighted mixture velocity);

- A mixture model with a volume fraction formulation and a diffusion of phases between particles following a Fick law has been implemented and successfully tested on gravity currents 
in Cueille (2005), extending to the Lagrangian framework the Eulerian model proposed by Chanteperdrix (2004);

- Following Cueille (2005), a mixture model with a volume fraction formulation without phase exchanges between particles was implemented and compared to a multi-fluid formulation in Grenier (2009), especially on a Rayleigh-Taylor instability case. It highlighted the diffusion of the interfaces triggered by the mixture model. The multi-fluid formulation of SPH proved to perform better on the cases tested.

In this paper, the physical and numerical formulations chosen for a new mixture model are presented. It is designed to be able to deal with high density ratio flows and versatile physics. Special attention is paid to the conservativity, realizability (respect of physical bounds) and accuracy of this resolution, issues that have raised some noticeable interest as in Rusche (2002). Then, numerical validation on several cases is detailed, with comparisons to reference solutions in the literature. The cases comprise an oil-water and an air-water separation cases, an analytical case similar to a two-phase mixture Poiseuille flow, a Rayleigh-Taylor instability case and a sand dumping case.

\section{Mathematical model}

Let us consider a two-phase flow (e.g. air bubbles rising or sediments falling within water). This section aims at detailing the notations and the mixture equations adopted in this work to model such a flow. We consider the control volum 2 presented in Figure 1. The presence of phases is taken into account through volume fractions, as illustrated schematically in Figure 2. The two phases are denoted by $\alpha$ and $\beta$.

\subsection{Notations}

The mixture quantities detailed in Table 2 are computed based on the value of some phase quantities listed in Table 1. Several choices are possible for the phase quantities to be considered: in view of achieving the conservation of phase quantities at high density ratios, a volume formulation has been retained here for the phase description (volume fraction 3 ) and mixture velocity. The

\footnotetext{
${ }^{2}$ The control volume models an SPH particle that will be introduced in the next section.

${ }^{3}$ We denote $\alpha$ and $\beta$ the volume fractions as well as the phase names, without any risk of confusion.
} 


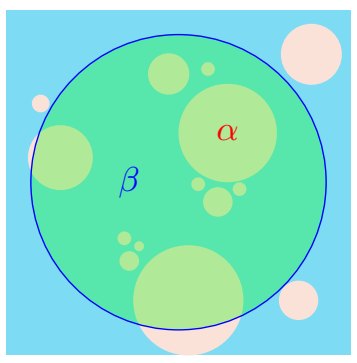

Figure 1: Control volume (green area) in two-phase flow (here a gas phase in red, liquid phase in blue).

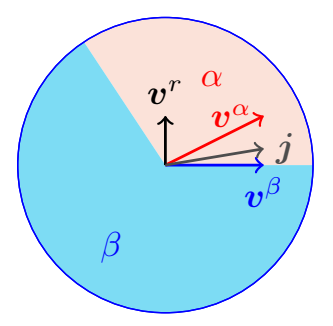

Figure 2: Schematic view of the control volume, the corresponding volume fractions and velocity fields.

mixture velocity is indeed defined here with respect to the volume centre, and is also called volumetric flux, due to weighting by volume fractions. A weighting by mass fractions would correspond to a definition with respect to the mass center. A mass formulation using mass fractions would lead to a simpler set of mixture equations but trigger some numerical issues for high density ratio flows. To preserve the symmetry of the system, we choose to work with relative velocities instead of drift/diffusion velocities.

\subsubsection{Mixture quantities}

As a consequence of the above-mentioned definitions, one has the following useful relations:

$$
\begin{gathered}
\alpha+\beta=1 \\
\boldsymbol{v}^{\alpha}=\boldsymbol{j}+\beta \boldsymbol{v}^{r} \quad \text { and } \quad \boldsymbol{v}^{\beta}=\boldsymbol{j}-\alpha \boldsymbol{v}^{r}
\end{gathered}
$$

The Lagrangian derivative for a particle moving at the mixture velocity $\boldsymbol{j}$ is denoted by:

$$
\frac{d}{d t}=\frac{\partial}{\partial t}+j \cdot \nabla
$$




\begin{tabular}{l|l}
\multicolumn{2}{c}{ Table 1: Phase quantities $(\mathrm{k}=\alpha$ or $\beta)$} \\
\hline \hline Volume & $V^{k}$ \\
Mass & $m^{k}$ \\
Density & $\rho^{k}=m^{k} / V^{k}$ \\
Kinematic viscosity & $\nu^{k}$ \\
Dynamic viscosity & $\mu^{k}$ \\
Velocity & $\boldsymbol{v}^{k}$ \\
Pressure & $p^{k}$ \\
\hline \hline
\end{tabular}

\subsection{Mixture model}

Following Ishii \& Hibiki (2011), the averaged model for two-phase flows gives the following set of continuous phase equations:

$$
\begin{gathered}
\frac{\partial \alpha \rho^{\alpha}}{\partial t}+\nabla \cdot\left(\alpha \rho^{\alpha} \boldsymbol{v}^{\alpha}\right)=0 \\
\frac{\partial \alpha \rho^{\alpha} \boldsymbol{v}^{\alpha}}{\partial t}+\nabla \cdot\left(\alpha \rho^{\alpha} \boldsymbol{v}^{\alpha} \otimes \boldsymbol{v}^{\alpha}\right)=-\boldsymbol{\nabla}\left(\alpha p^{\alpha}\right)+\boldsymbol{\nabla} \cdot\left(\alpha \boldsymbol{T}^{\alpha}\right)+\alpha \rho^{\alpha} \boldsymbol{g}
\end{gathered}
$$

where $\boldsymbol{T}^{\alpha}=\mu^{\alpha}\left(\boldsymbol{\nabla} \boldsymbol{v}^{\alpha}+{ }^{t} \boldsymbol{\nabla} \boldsymbol{v}^{\alpha}\right)$ stands for the viscous stress tensor and $\boldsymbol{g}$ the gravity vector. We neglected here the interfacial terms. Combining these relations and using the mixture model variables introduced in 2.1. one gets:

$$
\begin{gathered}
\frac{\partial \rho}{\partial t}+\boldsymbol{j} \cdot \boldsymbol{\nabla} \rho=-\rho \boldsymbol{\nabla} \cdot \boldsymbol{j}+\boldsymbol{\nabla} \cdot\left(\alpha \beta\left(\rho^{\beta}-\rho^{\alpha}\right) \boldsymbol{v}^{r}\right) \\
\frac{\partial \alpha \rho^{\alpha}}{\partial t}+\boldsymbol{j} \cdot \boldsymbol{\nabla}\left(\alpha \rho^{\alpha}\right)=-\alpha \rho^{\alpha} \boldsymbol{\nabla} \cdot \boldsymbol{j}-\boldsymbol{\nabla} \cdot\left(\alpha \rho^{\alpha} \beta \boldsymbol{v}^{r}\right)
\end{gathered}
$$

Under the hypothesis of constant phase densities (i.e. $\rho^{\alpha}=c s t$ and $\rho^{\beta}=c s t$ ), one gets the volume fraction equation:

$$
\frac{\partial \alpha}{\partial t}+\boldsymbol{j} \cdot \boldsymbol{\nabla} \alpha=-\boldsymbol{\nabla} \cdot\left(\alpha \beta \boldsymbol{v}^{r}\right)
$$

Summing this relation on both phases leads to a divergence-free mixture velocity field. This conservation equation, if we except the relative velocity term, is somewhat similar to the Volume of Fluid approach proposed by Hirt \& Nichols (1981) or the topological equation introduced in 7-equations models for two-phase flows as detailed in Baer \& Nunziato (1986), neglecting compressibility and 
Table 2: Mixture quantities

\begin{tabular}{l|l}
\hline \hline Volume & $V=V^{\alpha}+V^{\beta}$ \\
Mass & $m=m^{\alpha}+m^{\beta}$ \\
Density & $\rho=m / V=\alpha \rho^{\alpha}+\beta \rho^{\beta}$ \\
Volume fractions & $\alpha=V^{\alpha} / V$ and $\beta=V^{\beta} / V$ \\
Mass fractions & $Y^{\alpha}=\alpha \rho^{\alpha} / \rho$ and $Y^{\beta}=\beta \rho^{\beta} / \rho$ \\
Mixture velocity w.r.t. the volume center & $\boldsymbol{j}=\alpha \boldsymbol{v}^{\alpha}+\beta \boldsymbol{v}^{\beta}$ \\
Mixture velocity w.r.t. the mass center & $\boldsymbol{u}=Y^{\alpha} \boldsymbol{v}^{\alpha}+Y^{\beta} \boldsymbol{v}^{\beta}$ \\
Relative velocity & $\boldsymbol{v}^{r}=\boldsymbol{v}^{\alpha}-\boldsymbol{v}^{\beta}$ \\
Drift velocity & $\boldsymbol{v}^{\alpha}-\boldsymbol{j}$ \\
Diffusion velocity & $\boldsymbol{v}^{\alpha}-\boldsymbol{u}$ \\
Mixture pressure & $p$ \\
\hline \hline
\end{tabular}

thermodynamical effects. A volume fraction equation has been proposed in Chanteperdrix (2004) and includes additional terms due to compressibility effects that might be considered in further studies. In this work we choose to use the usual momentum equation using mixture velocities and densities for the sake of simplicity, as we focus on volume fraction and density resolution in a first attempt at deriving an SPH mixture model:

$$
\frac{d \boldsymbol{j}}{d t}=-\frac{1}{\rho} \nabla p+\frac{1}{\rho} \boldsymbol{\nabla} \cdot \boldsymbol{T}+\boldsymbol{g}
$$

where $\boldsymbol{T}=\rho \nu\left(\boldsymbol{\nabla} \boldsymbol{j}+{ }^{t} \boldsymbol{\nabla} \boldsymbol{j}\right)$ with $\nu=\alpha \nu^{\alpha}+\beta \nu^{\beta}$ the mixture kinematic molecular viscosity. Chanteperdrix (2004) indicates that there is no preferential argument to choose either a mixture kinematic or dynamic viscosity. The choice done here is motivated by a momentum equation derivation in this volume-based framework that makes a mixture kinematic viscosity appear naturally. An equation consistent with the volume-based formulation would require additional careful work (as explained in Ishii \& Hibiki (2011), including an additional viscous terms and a contribution of the form $\left.\boldsymbol{\nabla} \cdot\left(\alpha \beta \boldsymbol{v}^{r} \otimes \boldsymbol{v}^{r}\right)\right)$.

\subsection{State equation}

We assume the mechanical equilibrium of partial pressures as pressure relaxation time is generally small compared to other characteristic times of the flow, as illustrated in Labois (2008) in case 
of bubbly flows:

$$
p=p^{\alpha}=p^{\beta}
$$

In the standard WCSPH (Weakly Compressible SPH) approach, the mixture is considered as barotropic and the pressure is calculated based on the density values, using the Tait equation of state as reported in Cole (1948). We adapted the state equation to a two-phase mixture with a unit polytropic index (common assumption, as in Marrone et al. (2011) for example) and a sound speed computed following Grenier (2009). We linked the pressure to a ratio of volumes instead of densities:

$$
p=\left(\alpha \rho^{\alpha}\left(c^{\alpha}\right)^{2}+\beta \rho^{\beta}\left(c^{\beta}\right)^{2}\right)\left(\frac{V_{0}}{V}-1\right)+p_{B}
$$

where $V_{0}$ is the reference volume equal to $\delta r^{2}$ in $2 \mathrm{D}, \delta r^{3}$ in $3 \mathrm{D}, \delta r$ being the particle discretization. This formula reduces to a one-phase formulation for null or unit volume fraction: in single-phase $\mathrm{SPH}$, when particle masses are constant, the ratio of densities $\rho / \rho_{0}$ can be identified to the ratio of volumes $V_{0} / V$. In the following, if not specified, we assume that the background pressure $p_{B}$ is

null and sound speeds are chosen so as to ensure that $c^{k}>10 \max \left(v_{\max }, \sqrt{g H}\right)$ where $v_{\max }$ is the maximum mixture velocity and $H$ the maximum height of the flow under gravity. As in classical WCSPH, this ensures the compressibility effect is purely numerical and the sound speeds $c^{\alpha}$ and $c^{\beta}$ aim at ensuring relative density fluctuations within 1\%, as explained by Monaghan (1994).

\section{Numerical model}

The fluid domain $\Omega$ is discretized with a set of SPH fluid particles $\mathcal{F}$. The discrete value $A_{b}=A\left(\boldsymbol{r}_{b}, t\right)$ denotes the quantity $A$ at the particle position vector $\boldsymbol{r}_{b}$ and time $t$.

\subsection{SPH operators}

The continuous SPH interpolation is performed with the Wendland $\mathbf{C}_{2}$ kernel introduced in Wendland (1995) denoted $w$. For a pair of particles $(a, b), w_{a b}=w\left(\boldsymbol{r}_{a b}\right)$ where $\boldsymbol{r}_{a b}=\boldsymbol{r}_{a}-\boldsymbol{r}_{b}$ and the kernel gradient writes $\nabla w_{a b}=\nabla_{a} w\left(\boldsymbol{r}_{a}-\boldsymbol{r}_{b}\right)$. Unified Semi Analytical Wall (USAW) boundary conditions described in Ferrand et al. (2017) are used. In this framework, boundaries $\partial \Omega$ of the domain are meshed by segments $s \in \mathcal{S}$ of size $\delta r$ ( $\delta r$ being the initial interparticle spacing) connected at vertices $v \in \mathcal{V}$ as illustrated in Figure 3 . Those vertices are truncated fluid particles 


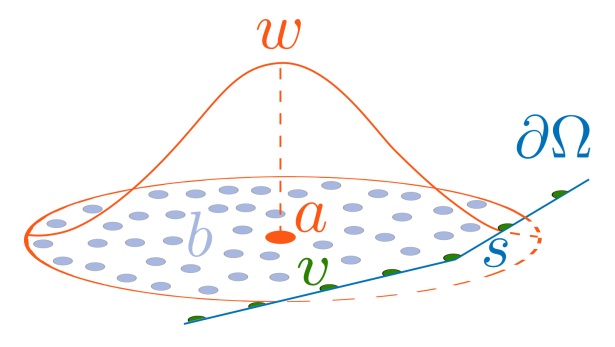

Figure 3: SPH kernel and intersecting boundary.

with a volume computed with respect to a reference volume through a fraction $\theta$. The reference volume for vertices and segments writes:

$$
\bar{V}_{a}=\frac{\sum_{b \in \mathcal{F}} V_{b} w_{a b}}{\sum_{b \in \mathcal{F}} w_{a b}}
$$

It is equal to the actual volume for fluid particles. On the other hand, $\theta$ is defined as the angle between two connected segments divided by $2 \pi$ in $2 \mathrm{D}$ for vertices, $1 / 2$ for segments and 1 for fluid particles. Segments and vertices contribute to accurately compute fields and their derivatives close to the boundaries through boundary and volume integrals, respectively. Vertices are indeed part of the fluid and allow one to impose field quantities. If not stated otherwise, multi-fluid operators adapted to this framework are employed following Ghaitanellis et al. (2015). The SPH pressure gradient approximation is therefore:

$$
\begin{aligned}
(\nabla p)_{a} \approx \boldsymbol{G}_{a}\left\{p_{b}\right\}= & \frac{1}{\gamma_{a} \bar{V}_{a}} \sum_{b \in \mathcal{F} \cup \mathcal{V}} \theta_{b}\left(\bar{V}_{a}^{2} p_{a}+\bar{V}_{b}^{2} p_{b}\right) \nabla w_{a b} \\
& -\frac{1}{\gamma_{a} \bar{V}_{a}} \sum_{s \in \mathcal{S}} \frac{1}{\bar{V}_{s}}\left(\bar{V}_{a}^{2} p_{a}+\bar{V}_{s}^{2} p_{s}\right) \nabla \gamma_{a s}
\end{aligned}
$$

In this operator, the renormalization factor $\gamma_{a}$ presented in Ferrand et al. (2017) was used to account for potential truncated kernel supports near boundaries:

$$
\gamma_{a}=\int_{\Omega_{a} \cap \Omega} w\left(\boldsymbol{r}_{a}-\boldsymbol{r}\right) d \boldsymbol{r}
$$

where $\Omega_{a}$ denotes the kernel support of the particle $a$. Moreover, following Ferrand et al. (2017), the contributions to the gradient of $\gamma$ are:

$$
\boldsymbol{\nabla} \gamma_{a s}=\left(\int_{s} w(r) d S\right) \boldsymbol{n}_{s}
$$


where $\boldsymbol{n}_{s}$ is the inward unit normal to the boundary segment $s$. These values can be computed analytically as explained in Ferrand et al. (2017).

Except in subsection 4.2, the SPH Laplacian approximation used to compute the viscous stress tensor writes:

$$
\begin{aligned}
(\boldsymbol{\nabla} \cdot(\mu \boldsymbol{\nabla} \boldsymbol{j}))_{a} \approx L_{a}\left\{\mu_{b}, \boldsymbol{j}_{b}\right\}= & \frac{1}{\gamma_{a}} \sum_{b \in \mathcal{F} \cup \mathcal{V}} \theta_{b} \bar{V}_{b} \frac{4 \mu_{a} \mu_{b}}{\mu_{a}+\mu_{b}} \frac{\boldsymbol{j}_{a b}}{r_{a b}^{2}} \boldsymbol{r}_{a b} \cdot \nabla w_{a b} \\
& -\frac{1}{\gamma_{a}} \sum_{s \in \mathcal{S}} 2 \mu_{a} \frac{\boldsymbol{j}_{a s}}{r_{a s}^{2}} \boldsymbol{r}_{a s} \cdot \nabla \gamma_{a s}
\end{aligned}
$$

where $r_{a b}=\left|\boldsymbol{r}_{a b}\right|, \boldsymbol{j}_{a b}=\boldsymbol{j}_{a}-\boldsymbol{j}_{b}$ and $\mu=\rho \nu$ being the dynamic molecular viscosity. Following Violeau (2009) and Español \& Revenga (2003), the alternative formulation used in case of variable viscosities is:

$$
\begin{aligned}
L_{a}\left\{\mu_{b}, \boldsymbol{j}_{b}\right\}= & \frac{1}{\gamma_{a}} \sum_{b \in \mathcal{F} \cup \mathcal{V}} \theta_{b} \bar{V}_{b} \frac{2 \mu_{a} \mu_{b}}{\mu_{a}+\mu_{b}}\left(4\left(\boldsymbol{j}_{a b} \cdot \boldsymbol{e}_{a b}\right) \boldsymbol{e}_{a b}+\boldsymbol{j}_{a b}\right) \frac{\boldsymbol{r}_{a b} \cdot \nabla w_{a b}}{r_{a b}^{2}} \\
& -\frac{1}{\gamma_{a}} \sum_{s \in \mathcal{S}} 2 \mu_{a} \frac{\boldsymbol{j}_{a s}}{r_{a s}^{2}} \boldsymbol{r}_{a s} \cdot \nabla \gamma_{a s}
\end{aligned}
$$

where $\boldsymbol{e}_{a b}=\frac{\boldsymbol{r}_{a b}}{r_{a b}}$. This formulation is used in subsection 4.2 .

\subsection{Integral balance for a single-phase flow}

The usual SPH discretisation of volume fraction equation (7) leads to non conservative and non realizable solutions (i.e. volume fractions may take non-physical values). We intend to derive a realizable phase volume equation, conservative with respect to the relative velocity contribution, through a finite-volume like approach. In this prospect, we will consider a Voronoi tesselation that discretizes the fluid, as illustrated in Figure 4. The cells in this Figure are a representation of the $\mathrm{SPH}$ particles, used to derive the subsequent equations. During a simulation, the exact shape of the particles is never actually known (or used). $a \mid b$ stands for the interface index between the cells $a$ and $b$, the outwards-oriented surface vector associated to this interface being denoted $\boldsymbol{S}_{a \mid b}$. For the sake of clarity, we first apply this approach to a single phase medium to see how one can recover the WCSPH volume conservation equation:

$$
\frac{d V}{d t}=V \nabla \cdot j
$$

After this, a similar reasoning shall be applied to the two-phase case. To start with, we only consider particles that do not interact with the boundaries of the domain. 


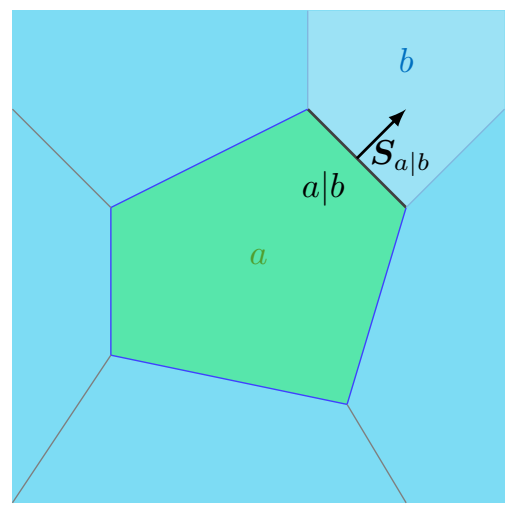

Figure 4: Voronoi diagram.

\subsubsection{Leibniz' rule}

Let us apply the Leibniz' rule for a scalar field $A$ on a particle $a$ seen as a material volume $\Omega_{a}$ moved and deformed by the fluid velocity $\boldsymbol{j}$ :

$$
\frac{d}{d t} \int_{\Omega_{a}(t)} A d V=\int_{\Omega_{a}(t)} \frac{\partial A}{\partial t} d V+\int_{\partial \Omega_{a}(t)} A \boldsymbol{j} \cdot \boldsymbol{d} \boldsymbol{S}
$$

For $A=1$, equation 19 gives:

$$
\frac{d V_{a}}{d t}=\int_{\partial \Omega_{a}(t)} \boldsymbol{j} \cdot \boldsymbol{d} \boldsymbol{S}
$$

We therefore recognize an integral form of equation (18). For a closed surface:

$$
\int_{\partial \Omega_{a}(t)} \boldsymbol{d S}=0
$$

Subtracting $\boldsymbol{j}_{a} \cdot 21$, one gets:

$$
\frac{d V_{a}}{d t}=\int_{\partial \Omega_{a}(t)}\left(\boldsymbol{j}-\boldsymbol{j}_{a}\right) \cdot \boldsymbol{d} \boldsymbol{S}
$$

\subsubsection{Discrete $S P H$ approximation}

Let us proceed with a finite volume formulation of the integrals in equation 22). For each neighbouring cell, one has:

$$
\begin{gathered}
\int_{a \mid b} d \boldsymbol{S}=\boldsymbol{S}_{a \mid b} \\
\int_{a \mid b} \boldsymbol{j} \cdot d \boldsymbol{S} \equiv \boldsymbol{j}_{a \mid b} \cdot \boldsymbol{S}_{a \mid b}
\end{gathered}
$$




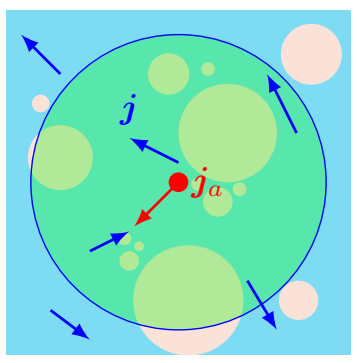

Figure 5: Mixture velocity field $\boldsymbol{j} . \boldsymbol{j}_{a}$ is the mixture velocity of the particle $a$, constant within the volume of the particle.

Equation 233 is exact while equation 24 is a definition of the interface mixture velocity $\boldsymbol{j}_{a \mid b}$. By summing over all neighbours (i.e. all the $a \mid b$ interfaces), one gets:

$$
\frac{d V_{a}}{d t}=\sum_{a \mid b}\left(\boldsymbol{j}_{a \mid b}-\boldsymbol{j}_{a}\right) \cdot \boldsymbol{S}_{a \mid b}
$$

In the above equation, $\boldsymbol{j}$ is the velocity at the continuous level, while $\boldsymbol{j}_{a}$ is the velocity of the particle $a$, which is a discrete field, constant over the support of the particle (see Figure 5).

The similarity between SPH-ALE and Finite Volume (FV) approaches have been underlined in Neuhauser (2015), among others. In the SPH formalism, this analogy is based on the following relation:

$$
\boldsymbol{S}_{a \mid b}=2 V_{a} V_{b} \boldsymbol{\nabla} w_{a b}
$$

to determine the virtual surface vector of the interface between particles. An important difference, as highlighted by Neuhauser (2015), between FV and SPH, is the definition of a neighbour in each method: only cells that share (part of) a face with the considered cell in FV are considered as neighbours, whereas all SPH particles within the kernel support are considered as neihgbours (their number thus depends on the smoothing length). This difference of neighbour definition requires an adequate handling of the weighting of the neighbouring SPH particle contribution, which is done through the presence of the kernel gradient in equation (26). A limit of this approach is that the property $\sum_{b} S_{a \mid b}=\mathbf{0}$, that is true in $\mathrm{FV}$, does not hold in SPH (as zeroth consistency is not fulfilled). This notation will be used throughout this document.

Choosing a centred value to approximate $\boldsymbol{j}_{a \mid b}$, equation 25$]$ becomes:

$$
\frac{d V_{a}}{d t}=-V_{a} \sum_{b \in \mathcal{F}} V_{b}\left(\boldsymbol{j}_{a}-\boldsymbol{j}_{b}\right) \cdot \nabla w_{a b}
$$


We recognize a discrete SPH approximation of (18), the right hand side being an SPH discrete divergence operator as described in Violeau (2012).

\subsection{Integral balance for a two-phase flow}

One can now make a similar reasoning in the case of a two-phase flow. Due to the mixture model we have adopted, we can consider that we have a single fluid, but whose characteristics will vary depending on the local volume fraction $\alpha$.

\subsubsection{Leibniz rule}

For $A=\alpha$, equation 19 becomes:

$$
\frac{d}{d t} \int_{\Omega_{a}(t)} \alpha d V=\int_{\Omega_{a}(t)} \frac{\partial \alpha}{\partial t} d V+\int_{\partial \Omega_{a}(t)} \alpha \boldsymbol{j} \cdot \boldsymbol{d} \boldsymbol{S}
$$

Using (8) to compute the right-hand side volume integral and using Gauss' theorem, we get:

$$
\frac{d V_{a}^{\alpha}}{d t}=\int_{\Omega_{a}(t)}\left[\alpha \boldsymbol{\nabla} \cdot \boldsymbol{j}-\boldsymbol{\nabla} \cdot\left(\alpha \beta \boldsymbol{v}^{r}\right)\right] d V
$$

Expanding $\alpha$ at the zeroth order, we make the following approximation:

$$
\int_{\Omega_{a}(t)} \alpha \boldsymbol{\nabla} \cdot \boldsymbol{j} d V=\alpha_{a} \int_{\Omega_{a}(t)} \boldsymbol{\nabla} \cdot \boldsymbol{j} d V
$$

Subtracting $\alpha_{a} \boldsymbol{j}_{a} \cdot 21$ leads to:

$$
\frac{d V_{a}^{\alpha}}{d t}=\alpha_{a} \int_{\partial \Omega_{a}(t)}\left(\boldsymbol{j}-\boldsymbol{j}_{a}\right) \cdot \boldsymbol{d} \boldsymbol{S}-\int_{\partial \Omega_{a}(t)} \alpha \beta \boldsymbol{v}^{r} \cdot \boldsymbol{d} \boldsymbol{S}
$$

\subsubsection{Discrete SPH approximation}

The finite volume approximation of the integrals leads to:

$$
\frac{d V_{a}^{\alpha}}{d t}=\alpha_{a} \sum_{a \mid b}\left(\boldsymbol{j}_{a \mid b}-\boldsymbol{j}_{a}\right) \cdot \boldsymbol{S}_{a \mid b}-\sum_{a \mid b}\left(\alpha \beta \boldsymbol{v}^{r}\right)_{a \mid b} \cdot \boldsymbol{S}_{a \mid b}
$$

where we identify two contributions: the first term corresponds to a divergence of the mixture velocity field $\alpha \boldsymbol{\nabla} \cdot \boldsymbol{j}$ and the second to a contribution from the relative displacements of phases $\boldsymbol{\nabla} \cdot\left(\alpha \beta \boldsymbol{v}^{r}\right)$. The interface $a \mid b$ quantities still need to be defined in equation (32). Four principles guide this definition:

- Conservativity: the fluxes must be symmetrical with respect to the particle labels $(a, b)$; 
- Realizability: phase volumes $V^{\alpha}$ and $V^{\beta}$ should remain positive;

- Reduction to single phase model: for $\alpha=1$ and $\boldsymbol{v}^{r}=\mathbf{0}$, we want to recover single-fluid WCSPH equations;

- Symmetry with respect to phases: we can interchange $\alpha \leftrightarrow \beta$ without modifying the equations.

Guided by these principles, we propose the following relations on both phase volumes:

$$
\begin{aligned}
& \frac{d V_{a}^{\alpha}}{d t}=\alpha_{a} \frac{d V_{a}}{d t}-2 V_{a} \sum_{b \in \mathcal{F}} V_{b}\left(\alpha_{a} \beta_{b}\left[\boldsymbol{v}_{a \mid b}^{r} \cdot \nabla w_{a b}\right]^{+}+\alpha_{b} \beta_{a}\left[\boldsymbol{v}_{a \mid b}^{r} \cdot \nabla w_{a b}\right]^{-}\right) \\
& \frac{d V_{a}^{\beta}}{d t}=\beta_{a} \frac{d V_{a}}{d t}+2 V_{a} \sum_{b \in \mathcal{F}} V_{b}\left(\alpha_{a} \beta_{b}\left[\boldsymbol{v}_{a \mid b}^{r} \cdot \nabla w_{a b}\right]^{+}+\alpha_{b} \beta_{a}\left[\boldsymbol{v}_{a \mid b}^{r} \cdot \nabla w_{a b}\right]^{-}\right)
\end{aligned}
$$

where $[x]^{+}=\max (x, 0)$ and $[x]^{-}=\min (x, 0)$. The relative velocity is computed with a centred approach:

$$
\boldsymbol{v}_{a \mid b}^{r}=\frac{1}{2}\left(\boldsymbol{v}_{a}^{r}+\boldsymbol{v}_{b}^{r}\right)
$$

Equation (34) was obtained from (33) by changing $\alpha$ into $\beta$, since these relations are symmetric (this switch changes the sign of the relative velocity). There are several important choices for the derivation of equation (33) from equation 32 :

- The first term of the right hand side of equation 32 can be identified as the first term of equation (33) using the relation (25). The computation of the temporal derivative of the total volume relies on the use of an exact time integration scheme, following what is suggested by Ferrand et al. (2017). It proves to be better at simulating the mixture at rest (the usual continuity equation resolution with antisymmetric divergence led to spurious convection cells which appeared to be linked to the accumulation of numerical errors). Starting from the SPH volume interpolation:

$$
\frac{\gamma_{a}}{V_{a}}=\sum_{b \in(\mathcal{F} \cup \mathcal{V})} \theta_{b} w_{a b}
$$

one can update the total volume through:

$$
\frac{\gamma_{a}^{n+1}}{V_{a}^{n+1}}-\frac{\gamma_{a}^{n}}{V_{a}^{n}}=\sum_{b \in(\mathcal{F} \cup \mathcal{V})}\left(\theta_{b}^{n+1} w_{a b}^{n+1}-\theta_{b}^{n} w_{a b}^{n}\right)
$$

- The second term of the right hand side of equation 32 is computed through an upwind formulation, symmetric with respect to phases, that ensures the realizability of the scheme 
(under a CFL condition, see section 3.7). The flux $\boldsymbol{v}_{a \mid b}^{r} \cdot \boldsymbol{S}_{a \mid b}$ at the midpoint of the pair of particles $(a, b)$ is split into its positive and negative parts. The factor $\alpha \beta$ then takes a different form depending on the sign of the flux: in $\alpha_{a} \beta_{b}, \alpha$ is upwinded with respect to $\boldsymbol{v}^{r}$ and $\beta$ is convected by $\left(-\boldsymbol{v}^{r}\right)$. The choice of alternate indices $a$ and $b$ for the volume fractions ensures the symmetry with respect to the phases and the conservation of the total quantity of each phase.

A similar formulation can be found in (Shi et al. 2017) that details a two-phase model for sediment laden flows with an asymmetric treatment of the liquid and solid phases. The volume fraction of sediment is updated with an equation looking like 33 but with $\beta$ taken to 1 and a usual SPH divergence of the fluid velocity field to compute the total volume variation. Instead of a $\beta$ equation, an equation on $\beta \rho^{\beta}$ is solved. Our approach has the advantage to keep a symmetrical treatment of both phases and to be conservative with respect to the relative velocity contribution. The model can therefore handle only water or air phase within a particle when the approach detailed in (Shi et al., 2017) assumes constant water mass in particles, which may trigger some significant volume variations. We are only solving three equations instead of four, which decreases the computational cost of the approach. A mixed upwind/downwind operator in a finite volume framework is proposed in Larreteguy et al. (2017) to solve a volume fraction equation with drift velocities and a massweighted mixture velocity ( $\alpha$ is upwinded whereas the drift velocity encompassing $\beta$ and $\boldsymbol{v}^{r}$ is downwinded). Such a distinction between upwind and downwind, due to a stencil limited to a pair of particles in SPH, does not seem possible to implement.

As in usual WCSPH, the total volume is not conserved due to weakly-compressibility. However, the antisymmetry of the discrete terms concerning the relative velocity ensures that the relative phase motion does not affect the total volume. Introducing the wall renormalization factor $\gamma$ of equation (14), the phase volume equation (33) becomes:

$$
\frac{d V_{a}^{\alpha}}{d t}=\alpha_{a} \frac{d V_{a}}{d t}-2 \frac{V_{a}}{\gamma_{a}} \sum_{b \in \mathcal{F}} V_{b}\left(\alpha_{a} \beta_{b}\left[\boldsymbol{v}_{a \mid b}^{r} \cdot \nabla w_{a b}\right]^{+}+\alpha_{b} \beta_{a}\left[\boldsymbol{v}_{a \mid b}^{r} \cdot \nabla w_{a b}\right]^{-}\right)
$$

the temporal variation of $V_{a}$ being computed from (37), the symmetry of the relative velocity term is somewhat broken by this factor, as always with the USAW technique but it did not prove to introduce significant discrepancies. No boundary term is considered so as to ensure a no-flux condition at the boundaries. Let us finally underline that in the numerical implementation phase volumes have been adimensionalized by the reference volume $V_{0}$ to avoid the accumulation of 
numerical errors that triggered some spurious pressure profiles.

\subsection{Volume diffusion}

For constant relative velocities, checkerboard effects clearly appear in simulations as a consequence of the colocated nature of SPH. One possible way to circumvent this issue is to introduce volume diffusion when phase volumes are updated. The derivation of this additional term is detailed in Appendix A.

\subsection{Particle characteristics}

Once phase volumes are computed, one can assess the other particle quantities according to their definitions (here particle labels $a$ were dropped for the sake of simplicity):

- $m=\left(\alpha \rho^{\alpha}+\beta \rho^{\beta}\right) V_{0}$

- $\rho=m / V$

- $\alpha=V^{\alpha} / V$

Special attention shall be paid to the particle mass computation. In single-fluid SPH, particle masses are usually constant (except in the Arbitrary Lagrangian-Eulerian approaches such as in Vila (1999)), so that they do not vary due to compressibility effects; only the density does. To recover this behaviour, we introduce the reference volume $V_{0}$ to compute particle masses, so that they only vary due the volume fraction variations. With this choice, we indeed have a varying density due to the fluid (weak) compressibility. This way of calculating the particle quantities constitutes a discrete approximation of the original continuous equations (6) and (8). To check that, one can deduce from (33) the discrete governing equations of the mixture density and volume fraction:

$$
\begin{aligned}
\frac{d \rho}{d t}= & -\frac{\rho_{a}}{V_{a}} \frac{d V_{a}}{d t} \\
& -\left(\rho^{\alpha}-\rho^{\beta}\right) \frac{V_{0}}{V_{a}^{2}} \sum_{b}\left(\alpha_{a} \beta_{b}\left[\boldsymbol{v}_{a \mid b}^{r} \cdot \boldsymbol{S}_{a \mid b}\right]^{+}+\alpha_{b} \beta_{a}\left[\boldsymbol{v}_{a \mid b}^{r} \cdot \boldsymbol{S}_{a \mid b}\right]^{-}\right) \\
\frac{d \alpha}{d t} & =-\frac{1}{V_{a}} \sum_{b}\left(\alpha_{a} \beta_{b}\left[\boldsymbol{v}_{a \mid b}^{r} \cdot \boldsymbol{S}_{a \mid b}\right]^{+}+\alpha_{b} \beta_{a}\left[\boldsymbol{v}_{a \mid b}^{r} \cdot \boldsymbol{S}_{a \mid b}\right]^{-}\right)
\end{aligned}
$$

the sum on the right hand side being an SPH approximation of $\boldsymbol{\nabla}\left(\alpha \beta \boldsymbol{v}^{r}\right)$, as previously explained. 


\subsection{Closure law for the relative velocity}

The closure law for the relative velocity is flow-dependent and one can refer to the extensive literature, for example (Ishii \& Hibiki, 2011), to find adequate closures depending on the twophase flow regime. The relations used in this article assume constant particle sizes. More complex models solving transport equations for particle diameters exist but we will focus on the simple closures described hereafter. Let us note $\boldsymbol{v}_{0}$ the rising velocity of a single, small spherical bubble of diameter $d_{p}$ within an infinite medium at rest under gravity, according to Stokes' law:

$$
\boldsymbol{v}_{0}=\frac{\left(\rho^{\alpha}-\rho^{\beta}\right) d_{p}^{2}}{18 \mu^{\beta}} \boldsymbol{g}
$$

Under the assumption that a local equilibrium can be reached over a short spatial length scale, one can write an algebraic expression of the relative velocity according to Manninen \& Taivassalo (1996). Several cases are presented in this work:

- A constant relative velocity:

$$
\boldsymbol{v}^{r}=\boldsymbol{v}_{0}
$$

- In Brethour \& Hirt (2009), the relative velocity is variable and linked to the pressure gradient through:

$$
\boldsymbol{v}^{r}=\frac{V_{p}}{K_{p}} \frac{\alpha\left(\rho^{\alpha}-\rho^{\beta}\right)}{\rho} \nabla p
$$

where the drag coefficient $K_{p}$ is computed as:

$$
K_{p}=\frac{1}{2} A_{p} \rho^{\beta}\left(C_{d}\left|\boldsymbol{v}^{r}\right|+\frac{24 \nu^{\beta}}{d_{p}}\right)
$$

with $A_{p}=\frac{1}{4} \pi d_{p}^{2}$ and $V_{p}=\frac{1}{6} \pi d_{p}^{3}$ the cross sections and volumes of the bubbles. To account for the particle-particle interactions, the Richardson-Zaki correlation can be employed to compute the effective relative velocity:

$$
\boldsymbol{v}_{\mathrm{eff}}^{r}=\alpha^{\zeta} \boldsymbol{v}^{r}
$$

where $\zeta$ is computed using the Reynolds number $R e=d_{p}\left|\boldsymbol{v}^{r}\right| / \nu^{\beta}$ as:

$$
\begin{cases}R e<1 & \zeta=4.35 / R e^{0.03} \\ 1<R e<500 & \zeta=4.45 / R e^{0.1} \\ 500<R e & \zeta=2.39\end{cases}
$$


To close 43, we use here $\nabla p=\rho \boldsymbol{g}$ in the separation case 4.1.1, assuming hydrostatic equilibrium.

- Finally, we propose a relative velocity including a volume fraction gradient:

$$
\boldsymbol{v}^{r}=\boldsymbol{v}_{0}-K \frac{\nabla \alpha}{\alpha}
$$

where the coefficient $K>0$ accounts for turbulent-like diffusion effects. The presence of a gradient requires some numerical precautions: to avoid double summation that can lead to instabilities, a second order SPH operator is used. Substituting (47) in (32), one gets:

$$
\frac{d V_{a}^{\alpha}}{d t}=\alpha_{a} \frac{d V_{a}}{d t}-\sum_{b \in \mathcal{F}}\left(\alpha \beta \boldsymbol{v}_{0}\right)_{a \mid b} \cdot \boldsymbol{S}_{a \mid b}+K \sum_{b \in \mathcal{F}}(\beta \boldsymbol{\nabla} \alpha)_{a \mid b} \cdot \boldsymbol{S}_{a \mid b}
$$

The relative velocity contribution in the phase volume equation is divided in two parts: the $\boldsymbol{v}_{0}$ part is treated as in (33) whereas the diffusion term in $K$ is discretized separately as a usual SPH Laplacian introduced by Morris et al. (1997):

$$
\begin{aligned}
& \frac{d V_{a}^{\alpha}}{d t}=\alpha_{a} \frac{d V_{a}}{d t}-\sum_{b \in \mathcal{F}}\left[\alpha_{a} \beta_{b}\left[\boldsymbol{v}_{0, a \mid b} \cdot \boldsymbol{S}_{a \mid b}\right]^{-}+\alpha_{b} \beta_{a}\left[\boldsymbol{v}_{0, a \mid b} \cdot \boldsymbol{S}_{a b}\right]^{+}\right] \\
& +K \sum_{b \in \mathcal{F}} \frac{\beta_{a}+\beta_{b}}{2} \frac{\alpha_{a}-\alpha_{b}}{r_{a b}} \frac{\boldsymbol{r}_{a b} \cdot \boldsymbol{S}_{a \mid b}}{r_{a b}}
\end{aligned}
$$

\subsection{CFL condition}

The resolution of the phase volume equation 33 introduces a condition on the time step for positivity of the phase volume (i.e. realizability of the numerical scheme). Let us consider the relative velocity of general form (47). It is associated to the numerical Péclet number

$$
P e_{\sigma}=\frac{v_{\max } \sigma}{K}
$$

where $v_{\max }=\max \left(\left|\boldsymbol{v}_{0}\right|\right)$ and $\sigma$ is the kernel standard deviation described in Violeau \& Leroy (2014). Appendix B details the derivation of the sufficient condition that guarantees the positivity of $V_{a}^{\alpha, n+1}$ given the positivity of $V_{a}^{\alpha, n}, V_{a}^{\beta, n}$ :

$$
C F L=\frac{v_{\max } \delta t}{\sigma} \leq \xi\left(\eta+\frac{1}{P e_{\sigma}}\right)^{-1}
$$

The non-positivity of $V^{\alpha}$ can trigger some instabilities so that this sufficient condition of positivity is interpreted as a condition of stability. For an advection-diffusion of the volume fraction $\alpha$ along the vertical axis, we got numerically $\xi \approx 1.1$ and $\eta \approx 0.8$ for $P e_{\sigma} \in[0.0012,0.12]$ (optimized with respect to the $\mathcal{L}_{1}$ norm of the relative error). The fitting curve is displayed in Figure 6. 


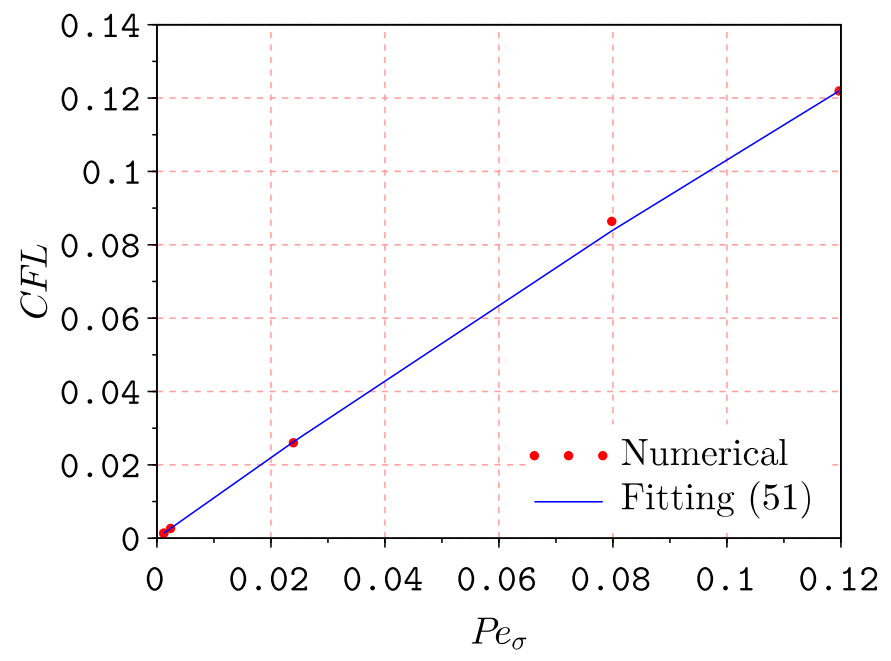

Figure 6: Advection-diffusion: limit of stability of the volume fraction profile (stability region below the curve).

\subsection{Boundary conditions}

In what follows, except in subsection 4.2 , the volume fraction at boundaries is computed with a Neumann condition using the following approximation:

$$
\alpha_{v}=\sum_{b \in \mathcal{F}} V_{b}^{\alpha} w_{a b}
$$

This value of the volume fraction of vertex particles is used in some of the operators described above.

\subsection{Time marching scheme}

The detailed resolution of our system is as follows (particle labels are dropped here):

1. Relative velocity update using 3.6 .

$$
\boldsymbol{v}^{r, n}=g\left(\rho^{n}, \alpha^{n}, \beta^{n}\right)
$$

2. Operator splitting for the momentum equation: velocity update

- Potential force step:

$$
\boldsymbol{j}^{*}=\boldsymbol{j}^{n}+\delta t\left[-\frac{1}{\rho^{n}} \boldsymbol{\nabla} p^{n}+\boldsymbol{g}\right]
$$


- Viscous force step:

$$
\boldsymbol{j}^{n+1}=\boldsymbol{j}^{*}+\delta t\left[\frac{1}{\rho^{n}} \boldsymbol{\nabla} \cdot \boldsymbol{T}^{*}\right] \text { with } \boldsymbol{T}^{*}=\rho^{n} \nu^{n}\left(\boldsymbol{\nabla} \boldsymbol{j}^{*}+{ }^{t} \boldsymbol{\nabla} \boldsymbol{j}^{*}\right)
$$

3. Particle position update:

$$
\boldsymbol{r}^{n+1}=\boldsymbol{r}^{n}+\delta t \boldsymbol{j}^{n+1}
$$

4. Total volume update using (37):

$$
V^{n+1}=f\left(V^{n}, \boldsymbol{r}^{n}, \boldsymbol{r}^{n+1}\right)
$$

5. Phase volume computations with (33) and (34):

$$
\begin{aligned}
& V^{\alpha, n+1}=V^{\alpha, n}+\alpha^{n}\left(V^{n+1}-V^{n}\right)-\delta t V^{n} \boldsymbol{\nabla} \cdot\left(\alpha^{n} \beta^{n} \boldsymbol{v}^{r, n}\right) \\
& V^{\beta, n+1}=V^{\beta, n}+\beta^{n}\left(V^{n+1}-V^{n}\right)+\delta t V^{n} \boldsymbol{\nabla} \cdot\left(\alpha^{n} \beta^{n} \boldsymbol{v}^{r, n}\right)
\end{aligned}
$$

6. Computation of other flow features using 3.5 .

$$
m^{n+1}, \rho^{n+1}, \alpha^{n+1}
$$

7. Pressure computation using state equation (11):

$$
p^{n+1}=p\left(\alpha^{n+1}, \beta^{n+1}, \rho^{n+1}\right)
$$

To comply with the saturation condition (1), we deduce $\beta=1-\alpha$ when needed in the implementation.

\section{Numerical validation}

All our application cases are two-dimensional with particles initially placed on a Cartesian lattice.

\subsection{Separation}

The separation of liquid dispersions, the sedimentation in a settling tank, etc. are common industrial separation problems. In this subsection, we investigate two applications of this separation phenomenon illustrated in Figure 7. This test case is of particular interest to check the realizability and conservativity of the numerical scheme. 


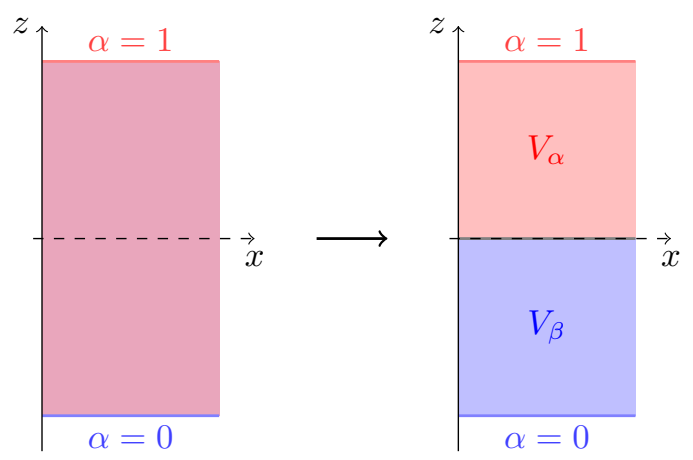

Figure 7: Separation case: initial and final states.

\subsubsection{Separation of an oil-water dispersion}

A vertical rectangular column $(0.24 \mathrm{~m} \times 0.915 \mathrm{~m})$ contains a mixture of oil of density $\rho^{\alpha}=837$ $\mathrm{kg} / \mathrm{m}^{3}$ and demineralized water of density $\rho^{\beta}=996 \mathrm{~kg} / \mathrm{m}^{3}$, with an initial uniform volume fraction of oil $\alpha_{0}=0.3$. The kinematic viscosities $\nu^{\alpha}=10^{-6} \mathrm{~m}^{2} / \mathrm{s}$ and $\nu^{\beta}=1.510^{-6} \mathrm{~m}^{2} / \mathrm{s}$ are used for the relative velocity computation. Sound speeds are set as $c^{\alpha}=c^{\beta}=45 \mathrm{~m} / \mathrm{s}$. The particle discretization is $\delta r=0.005 \mathrm{~m}$ to have an accurate tracking of the interface position. In the momentum equation, in order to stabilize the flow, we used higher viscosities, equal for both fluids $\nu^{\alpha}=\nu^{\beta}=0.03$ $\mathrm{m}^{2} / \mathrm{s}$. As the mixture is assumed to remain in hydrostatic equilibrium, this should not influence the result very much: particles have negligible motions and the separation is driven by the phase volume equation that triggers phase exchanges between particles. Experimental and numerical validation (Flow-3D ${ }^{\circledR}$ software) are provided in Jeelani \& Hartland (1998) and Brethour \& Hirt (2009), respectively. Flow-3 $\mathrm{D}^{\circledR}$ is a finite difference code based on structured meshes as described in Freitas (1995). It uses a semi-implicit resolution of the continuity/momentum equation with a first order convergence. For dispersed two-phase flow, it provides a mixture model similar to the one exhibited in this paper with a volume-based formulation using a volume-weighted mixture velocity (to automatically enforce incompressibility). This model together with the closure of the relative velocity are described in Brethour \& Hirt (2009) and detailed in 43. A switch of the definition of the dispersed phase is done at the threshold $\alpha=0.5$, so that continuous and dispersed phase are exchanged in the relative velocity definition. The relative velocity parameters are $d_{p}=1.2 \mathrm{~mm}$ and $C_{d}=0.7$ (values used in Flow-3D ${ }^{\circledR}$ ). The temporal evolution of the separation is displayed in Figure 8. We can observe the generation and motion of two clear interfaces until 




(a) $\mathrm{t}=0 \mathrm{~s}$

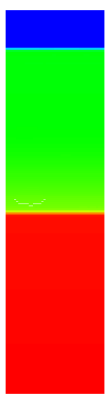

(b) $\mathrm{t}=50 \mathrm{~s}$

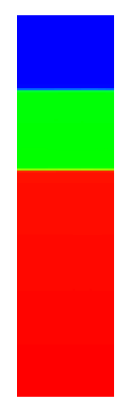

(c) $\mathrm{t}=100 \mathrm{~s}$

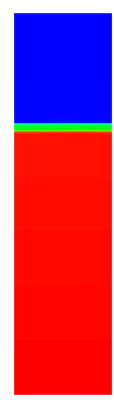

(d) $\mathrm{t}=150 \mathrm{~s}$
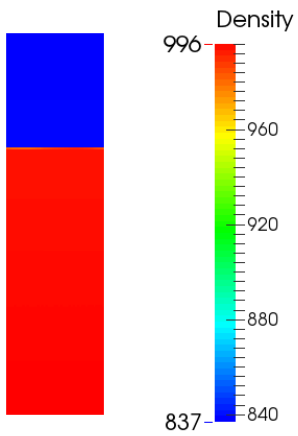

(e) $\mathrm{t}=200 \mathrm{~s}$

Figure 8: Separation of an oil-water dispersion: density evolution through the decantation process.

complete separation of oil and water phases. Figure 9 shows the evolution of the positions of these interfaces through the separation process, and shows good agreement with Flow-3D ${ }^{\circledR}$ and experimental results. Convergence is illustrated by the final interface position, located at $30 \%$ to the top wall. The slowdown of the interface progression velocity around $50 \mathrm{~s}$ is reproduced, and appeared to be linked to the switch of definitions of dispersed and continuous phases made at $\alpha=0.5$ for the computation of the relative velocity.

\subsubsection{Separation of an air-water mixture}

A vertical rectangular column $(1 \mathrm{~m} \times 2 \mathrm{~m})$ contains a mixture of air of density $\rho^{\alpha}=1.23 \mathrm{~kg} / \mathrm{m}^{3}$ and water of density $\rho^{\beta}=1000 \mathrm{~kg} / \mathrm{m}^{3}$, with an initial volume fraction of air $\alpha_{0}=0.5$. As a first step, high kinematic viscosities $\nu^{\alpha}=\nu^{\beta}=0.03 \mathrm{~m}^{2} / \mathrm{s}$ are taken. Sound speeds are $c^{\alpha}=c^{\beta}=45 \mathrm{~m} / \mathrm{s}$. The particle discretization is $\delta r=0.01 \mathrm{~m}$. The relative velocity is set to $0.45 \mathrm{~m} / \mathrm{s}$. Figure 10(a) shows that at convergence, both fluids are separated, and volume is conserved (the interface appears in the middle of the domain). Once the separation is established, we observe some instabilities developing near the interface, due to the pressure gradient computation, as highlighted in Figure 10(b). The pressure gradient discontinuity is however well computed, as shown in Figure 11(a), One can see in Figure 11(b) that there is a non negligible error at the interface, but also near the upper boundary as pressure values are quite small (for the relative error computation, the minimum pressure in the domain has been subtracted, as a constant pressure offset was present throughout the domain). In the bulk of the fluid domain, the error is close to 1\%, as expected in WCSPH. 


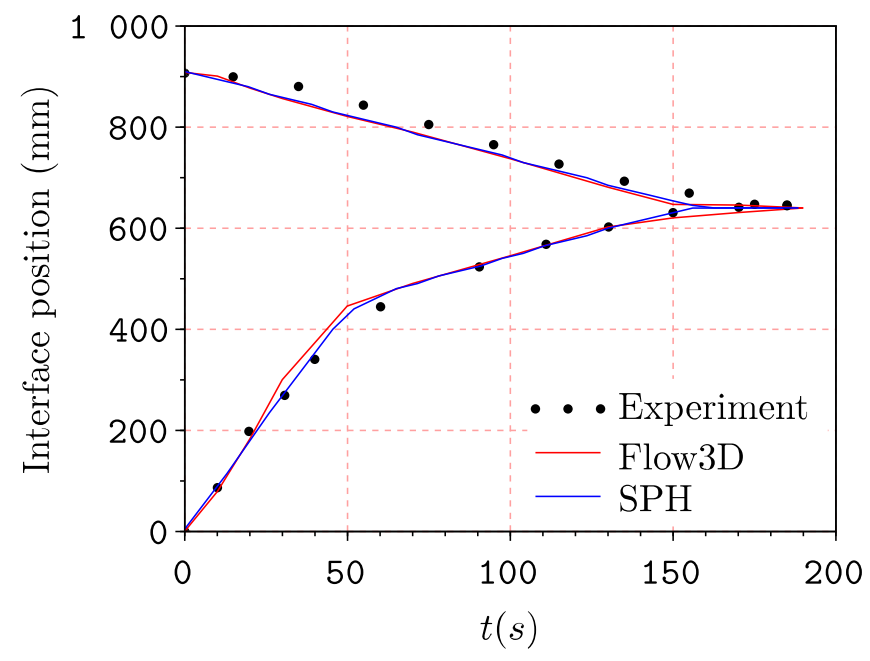

Figure 9: Separation of an oil-water dispersion: evolution of the upper and lower interface positions. Comparison of experimental data of Jeelani \& Hartland (1998) and numerical results (Flow-3D ${ }^{\circledR}$ results are taken from Brethour \& Hirt (2009)).

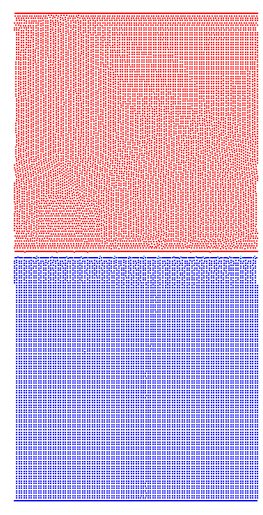

(a) Global view

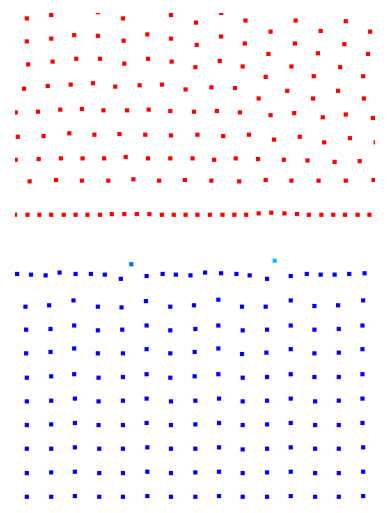

(b) Focus on the interface

Figure 10: Separation of an air-water mixture: separated state. 


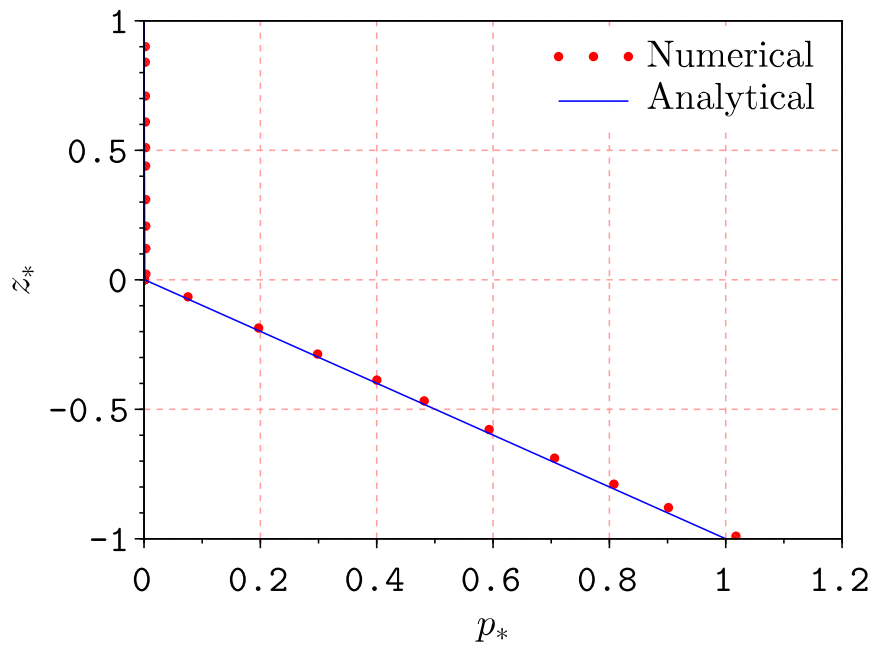

(a) Pressure profile

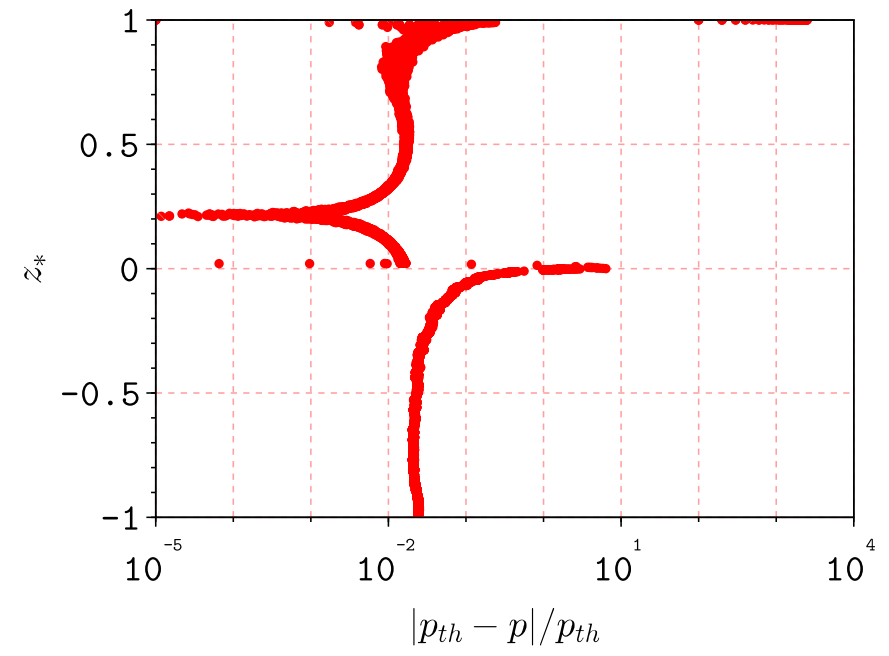

(b) Relative error

Figure 11: Separation of an air-water mixture: pressure for the separated state.

\subsection{Two-phase mixture Poiseuille flow}

The computation domain is a rectangle of height $2 e$ and length $e$, periodic along the $x$ direction as illustrated in Figure 12. The fluid is submitted to a longitudinal force $F$ mimicking a pressure gradient as in a Poiseuille flow. However, no gravity is introduced as it proved to trigger some small instabilities in the velocities and pressure profiles (the gravity effect is all included in the definition of the relative velocity). Starting from an homogeneous mixture $\alpha=\alpha_{0}$ at rest, the flow should converge towards the analytical dimensionless steady state detailed in Appendix C, obtained with a relative velocity given by (47) at constant mixture kinematic viscosity $\nu^{\alpha}=\nu^{\beta}$. The volume fraction at vertices $v$ of normal $\boldsymbol{n}_{v}$ is computed thanks to a second order approximation method for Robin boundary condition as detailed in Mayrhofer et al. (2013). The no-flux condition at the boundary:

$$
\boldsymbol{v}^{r}=\mathbf{0} \quad \longleftrightarrow \quad \alpha\left(\boldsymbol{v}_{0} \cdot \boldsymbol{n}\right)-\frac{\partial \alpha}{\partial \boldsymbol{n}}=0
$$

\footnotetext{
${ }^{4}$ Obtained with an incompressibility hypothesis.
} 


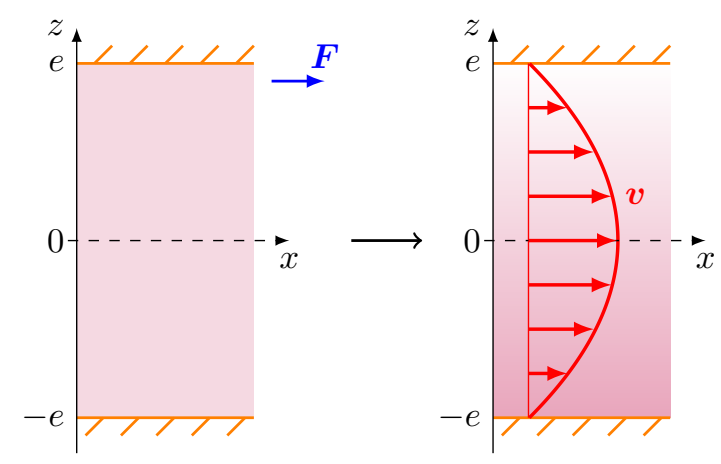

Figure 12: Two-phase mixture Poiseuille flow: initial and final states.

where $\boldsymbol{n}$ is the normal to the wall is approximated using:

$$
\begin{aligned}
\alpha_{v} & =\sum_{b \in \mathcal{F}} \frac{V_{b}^{\alpha}}{\Delta_{v}}\left(Y_{v}\left(1-\left(\boldsymbol{v}_{0} \cdot \boldsymbol{n}_{v}\right)\left(\boldsymbol{r}_{v b} \cdot \boldsymbol{n}_{v}\right) / K\right)-Z_{v}\left(\boldsymbol{r}_{v b} \cdot \boldsymbol{n}_{v}\right)^{2}\right) w_{v b} \\
\Delta_{v} & =X_{v} Y_{v}-Z_{v}^{2} \\
X_{v} & =\sum_{b \in \mathcal{F}} V_{b}\left(1-\left(\boldsymbol{v}_{0} \cdot \boldsymbol{n}_{v}\right)\left(\boldsymbol{r}_{v b} \cdot \boldsymbol{n}_{v}\right) / K\right)^{2} w_{v b} \\
Y_{v} & =\sum_{b \in \mathcal{F}} V_{b}\left(\boldsymbol{r}_{v b} \cdot \boldsymbol{n}_{v}\right)^{4} w_{v b} \\
Z_{v} & =\sum_{b \in \mathcal{F}} V_{b}\left(1-\left(\boldsymbol{v}_{0} \cdot \boldsymbol{n}_{v}\right)\left(\boldsymbol{r}_{v b} \cdot \boldsymbol{n}_{v}\right) / K\right)\left(\boldsymbol{r}_{v b} \cdot \boldsymbol{n}_{v}\right)^{2} w_{v b}
\end{aligned}
$$

This flow is analogous to a Rouse flow described in Rouse (1937) but with a rising instead of settling velocity and constant turbulent coefficient $K$. The following parameters were used: $e=1$ $\mathrm{m}, \rho^{\alpha}=1.23 \mathrm{~kg} / \mathrm{m}^{3}, \rho^{\beta}=1000 \mathrm{~kg} / \mathrm{m}^{3}, \nu^{\alpha}=\nu^{\beta}=0.03 \mathrm{~m}^{2} / \mathrm{s}$. Sound speeds are $c^{\alpha}=c^{\beta}=1$ $\mathrm{m} / \mathrm{s}$. Background pressure is set to $p_{B}=250 \mathrm{~Pa}$ to avoid particle disorder near boundaries that usually appears in Poiseuille flows. The particle discretization is $\delta r=0.01 \mathrm{~m}$. Initially starting with a uniform volume fraction distribution $\alpha_{0}=0.05$ and a fluid at rest, for $F=0.02 \mathrm{~N}$ and $P e=7.26\left(K=0.1 \mathrm{~m}^{2} / \mathrm{s}\right.$ and $d_{p}=0.2 \mathrm{~m}$, values chosen arbitrarily to exhibit a steep volume fraction gradient), one gets, after convergence, volume fraction and longitudinal velocity profiles displayed in Figures 13 and 14 . There is a very good agreement between analytical and numerical solutions. Let us underline that the highest relative errors are obtained in zones where the volume fraction or the mixture velocity are very small.

Convergence curves are displayed for the volume fraction and longitudinal velocity profiles in Figures 15 and 16 respectively, plotting the instantaneous $\mathcal{L}^{2}$ relative error for the steady-state field 


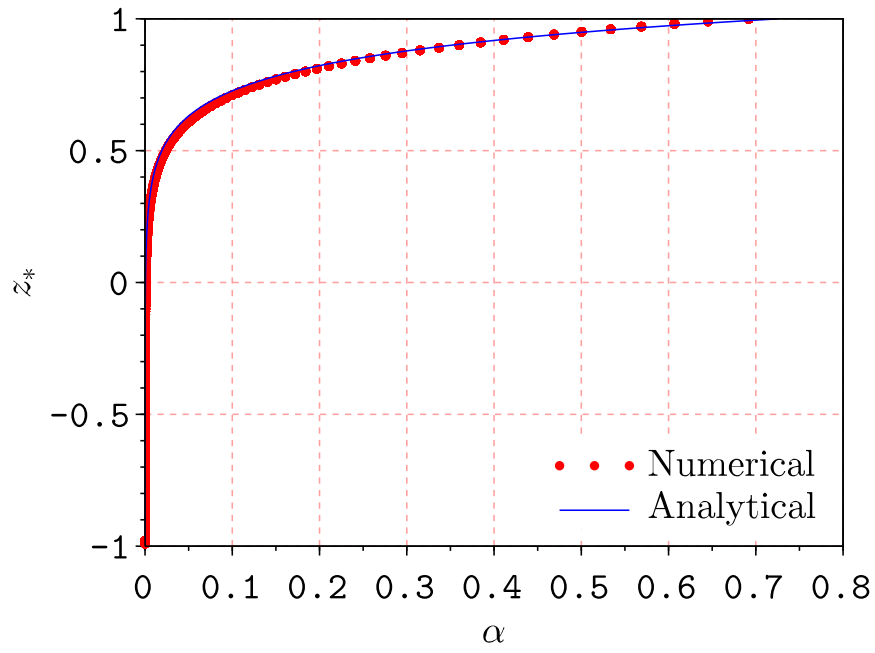

(a) Volume fraction profile

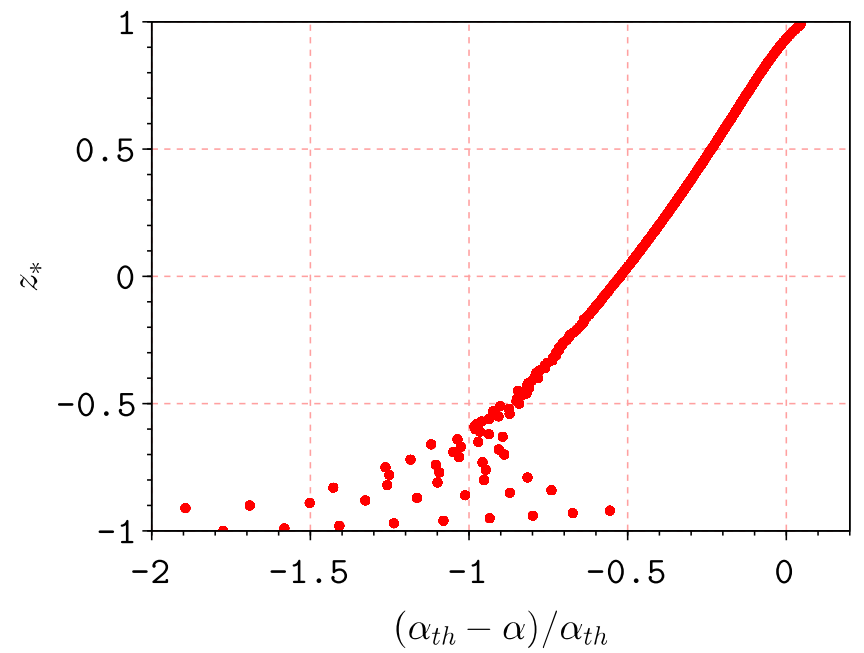

(b) Relative error

Figure 13: Two-phase mixture Poiseuille flow with constant kinematic viscosity: volume fraction at steady-state.

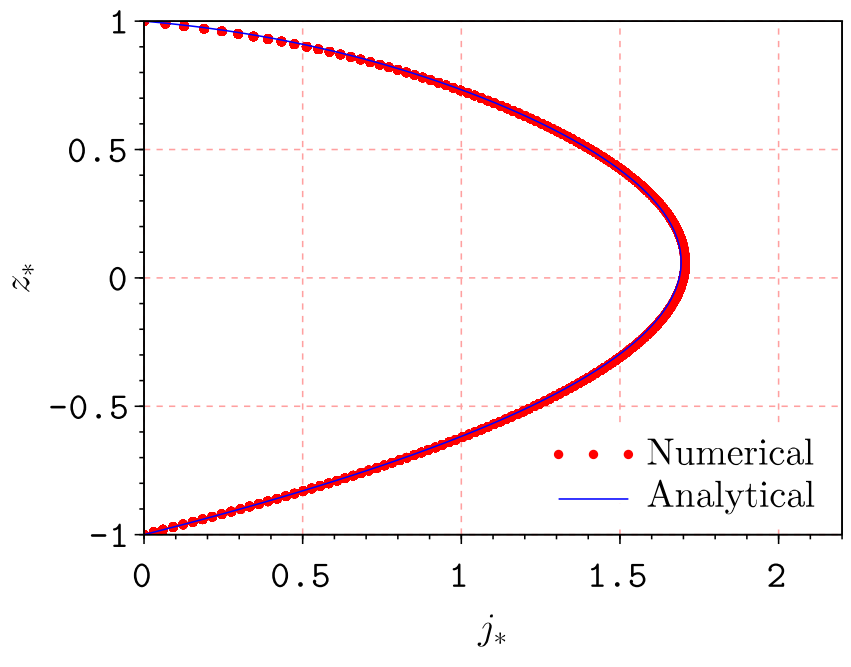

(a) Longitudinal velocity profile

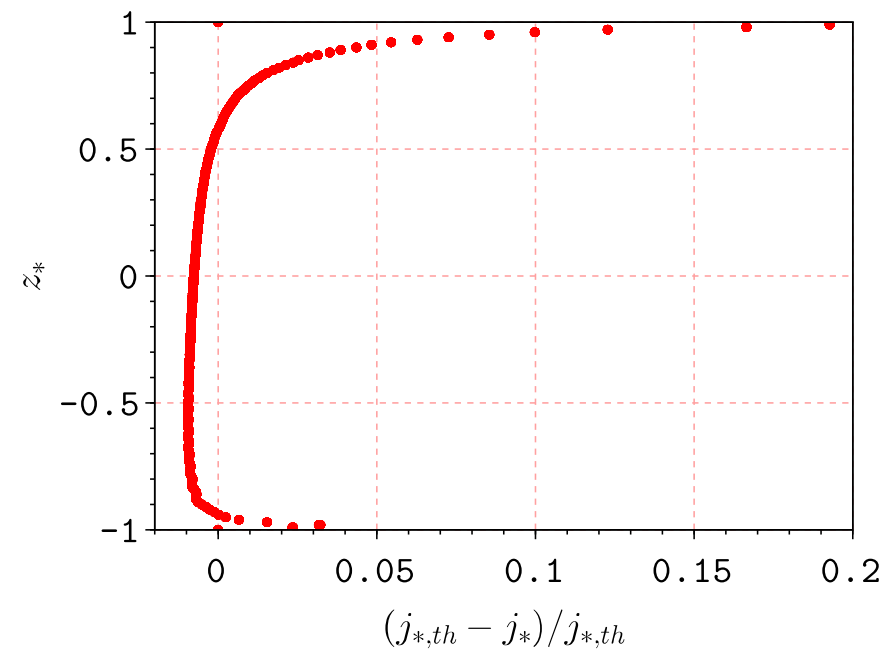

(b) Relative error

Figure 14: Two-phase mixture Poiseuille flow with constant kinematic viscosity: longitudinal velocity at steady-state. 


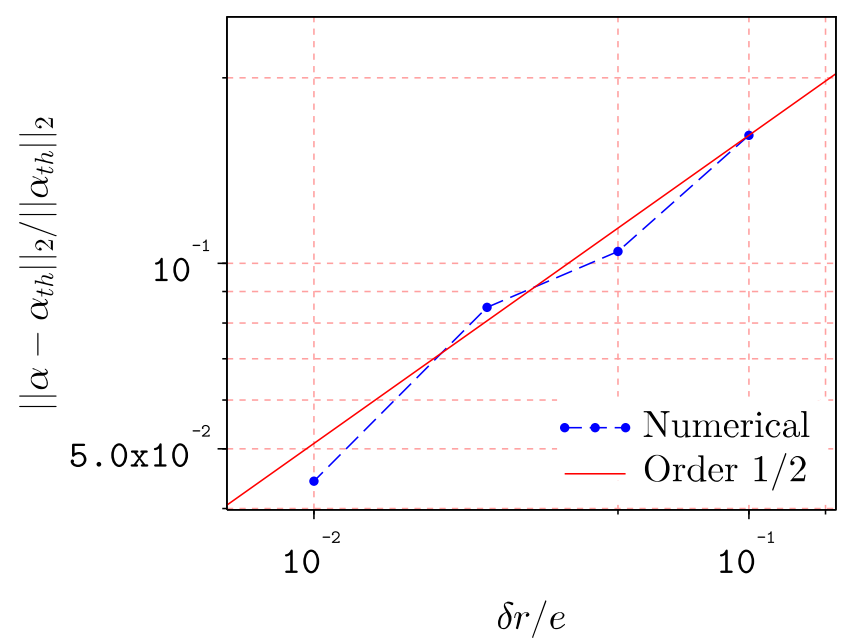

Figure 15: Two-phase mixture Poiseuille flow: convergence study on the volume fraction profile.

as a function of the dimensionless particle size $\delta r / e$. This relative error for a field $A$ compared to the analytical profile $A_{t h}$ is computed as:

$$
\frac{\left\|A-A_{t h}\right\|_{2}}{\left\|A_{t h}\right\|_{2}}=\sqrt{\frac{\sum_{b \in \mathcal{F}}\left(A_{b}-A_{t h}\left(z_{b}\right)\right)^{2}}{\sum_{b \in \mathcal{F}} A_{t h}\left(z_{b}\right)^{2}}}
$$

The convergence slope is approximately of order $1 / 2$ for the volume fraction and 1 for the longitudinal velocity. The upwind formulation of the phase volume equation might explain this weak order of convergence for the volume fraction.

As a second step, one can consider a case with a varying mixture kinematic viscosity. Only the longitudinal velocity profile is modified: its expression is detailed in Appendix C All parameters remain unchanged compared to previous configuration, except the viscosity $\nu^{\alpha}=0.001 \mathrm{~m}^{2} / \mathrm{s}$. We get, after convergence, the longitudinal velocity profile displayed in Figure 17 (the volume fraction profile is unchanged). Again, analytical and numerical solutions agree very well, even though, due to slight discrepancies on the volume fraction profiles, one can notice that the velocities are a bit smaller than expected in the upper part of the flow (the kinematic viscosity also varies with the volume fraction in this case, and the largest absolute errors on the volume fraction are made in the upper part of the flow, therefore influencing the shear stress, and consequently the quality of the 


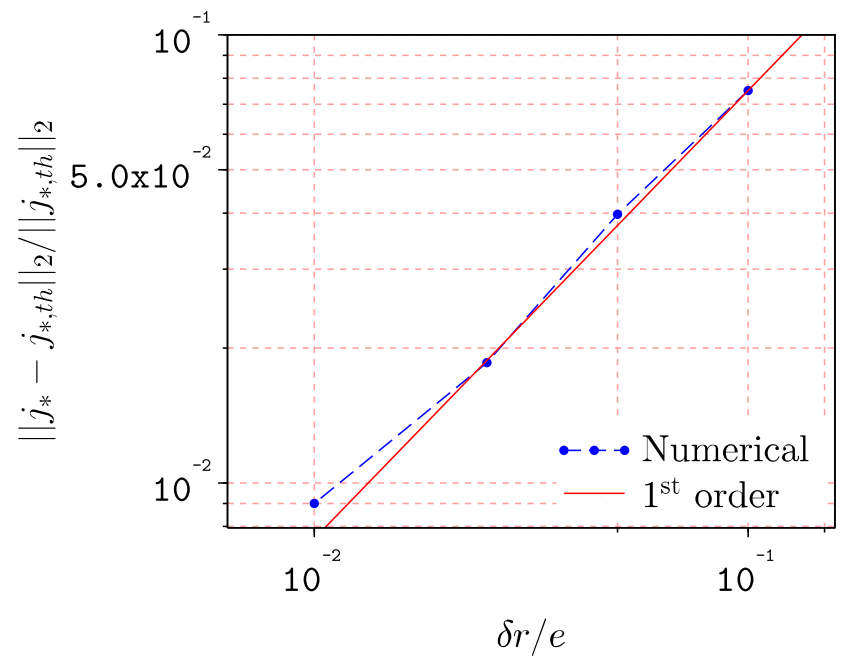

Figure 16: Two-phase mixture Poiseuille flow: convergence study on the longitudinal velocity profile.

velocity profile).

\subsection{Rayleigh-Taylor instability}

The computation domain is a rectangle of height $2 e$ and length $e$ bounded by walls. As illustrated in Figure 18, the fluid domain initially consists of two fluids of densities $\rho^{\alpha}=1000 \mathrm{~kg} / \mathrm{m}^{3}$ in the lower part and $\rho^{\beta}=1800 \mathrm{~kg} / \mathrm{m}^{3}$ in the upper part, separated by an interface defined by $z=1-0.15 \sin (2 \pi x)$. To be consistent with the work of Grenier et al. (2009), both fluids have the same kinematic viscosities $\nu=\nu^{\alpha}=\nu^{\beta}=2.38110^{-3} \mathrm{~m}^{2} / \mathrm{s}$ and the gravitational acceleration is taken to $g=1 \mathrm{~m} / \mathrm{s}^{2}$. The homogeneous model is solved, i.e. without relative velocities. To compare with the results from Grenier et al. (2009), a background pressure $p_{B}=3.6 \mathrm{~Pa}$ is introduced within a state equation of the form:

$$
p=\frac{\left(\alpha \rho^{\alpha}\left(c^{\alpha}\right)^{2}+\beta \rho^{\beta}\left(c^{\beta}\right)^{2}\right)}{\gamma}\left[\left(\frac{\rho}{\alpha \rho^{\alpha}+\beta \rho^{\beta}}\right)^{\gamma}-1\right]+p_{B}
$$

where $\gamma=7$ is the polytropic index. Sound speeds are $c^{\alpha}=14 \mathrm{~m} / \mathrm{s}$ and $c^{\beta}=10 \mathrm{~m} / \mathrm{s}$ to comply with the idea used for example in (Colagrossi \& Landrini, 2003) that one should choose values such that $\rho^{\alpha}\left(c^{\alpha}\right)^{2}=\rho^{\beta}\left(c^{\beta}\right)^{2}$. As spurious fragmentation occurs at the interface between fluids, a small repulsive force was added in the pressure gradient, following Grenier et al. (2009). This force 


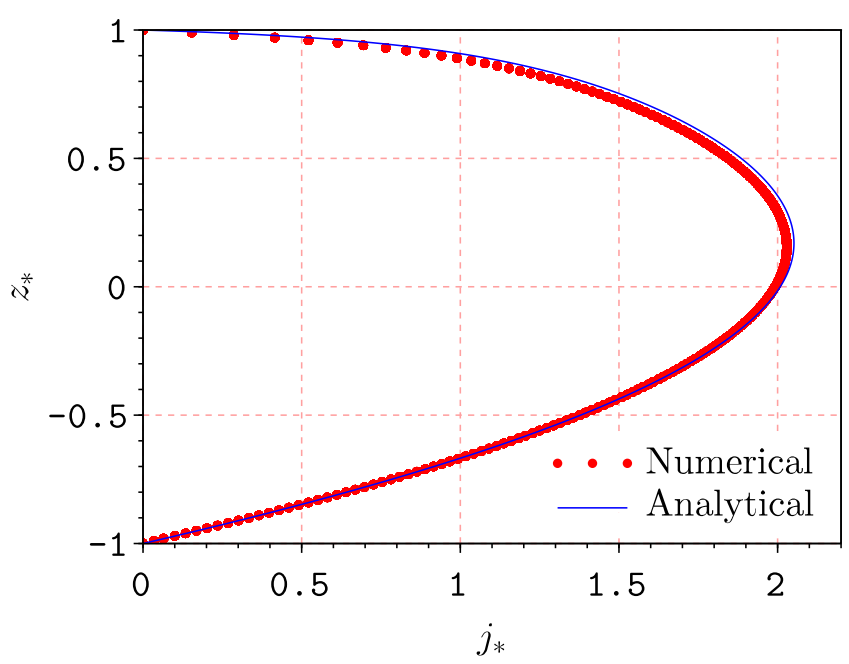

(a) Longitudinal velocity profile

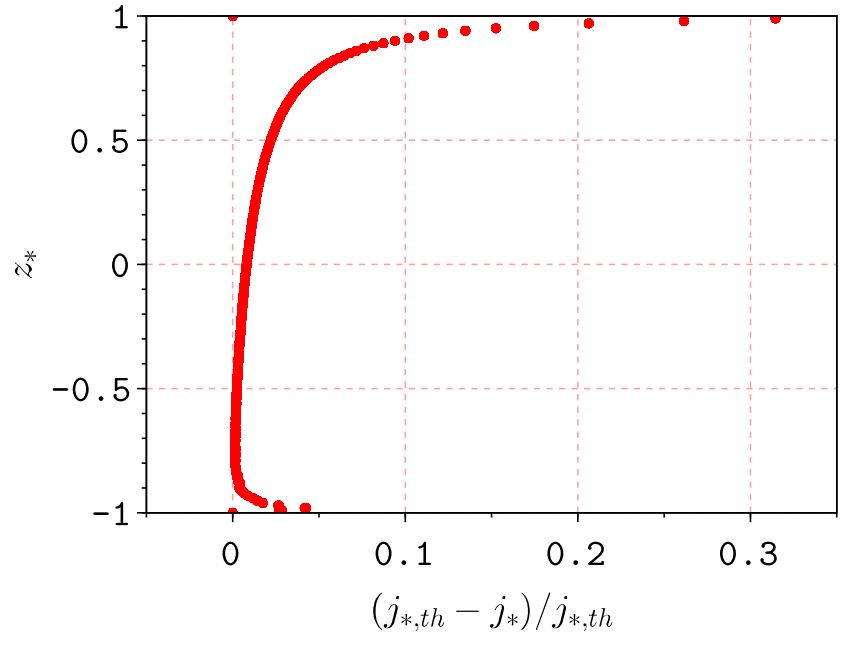

(b) Relative error

Figure 17: Two-phase mixture Poiseuille flow with varying kinematic viscosity: longitudinal velocity profile at steady-state.

consists of an additional term in the pressure gradient when the neighbouring particle belongs to another phase. Its expression was extended to the mixture model:

$$
\begin{aligned}
& \boldsymbol{G}_{a}\left\{p_{b}\right\}=\frac{1}{\gamma_{a} V_{a}} \sum_{b \in \mathcal{F}}\left(V_{a}^{2} p_{a}+V_{b}^{2} p_{b}\right) \nabla w_{a b} \\
& +\epsilon \frac{1}{\gamma_{a} V_{a}} \sum_{b \in \mathcal{F}}\left(\alpha_{a} \beta_{b}+\alpha_{b} \beta_{a}\right)\left(V_{a}^{2}\left|p_{a}\right|+V_{b}^{2}\left|p_{b}\right|\right) \nabla w_{a b}
\end{aligned}
$$

where the factor $\left(\alpha_{a} \beta_{b}+\alpha_{b} \beta_{a}\right)$ is equal to 1 when $a$ and $b$ belong to distinct phases, and 0 otherwise as long as phases remain separated, as in this Rayleigh-Taylor case without relative velocity. The relevance of this term for intermediate volume fractions is questionable. As suggested by Grenier et al. (2009), $\epsilon$ is taken equal to 0.01 . This test case aims at checking the numerical behaviour for separated phases: the multi-fluid behaviour should be recovered. Figure 19 shows the temporal evolution of the phases with the finest resolution (300 particles per unit length). Figure 20(a) displays a convergence study of the shape of the interface at the adimensional time $t_{*}=t \sqrt{g / e}=5$ in which one can see the convergence of the interface shape. Figure 20(b) compares the finest discretization interface with the one obtained in Grenier et al. (2009) with SPH and a Level-Set model at equivalent discretizations, showing a good agreement. Some discrepancies can be observed 


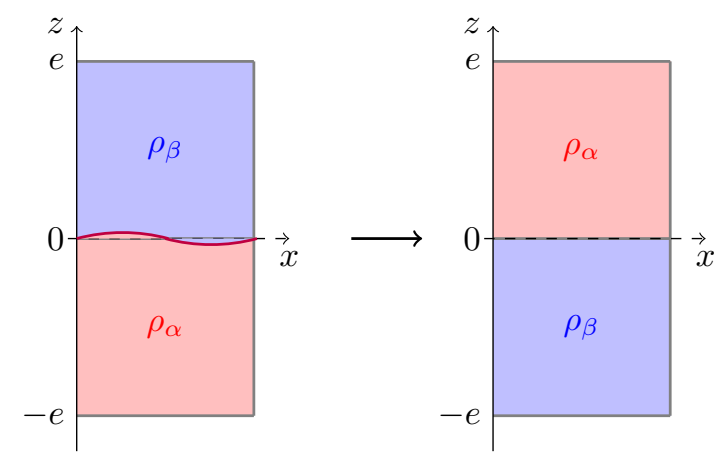

Figure 18: Rayleigh-Taylor instability: initial and final states.

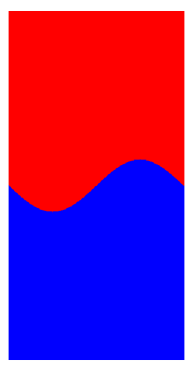

(a) $t_{*}=0$

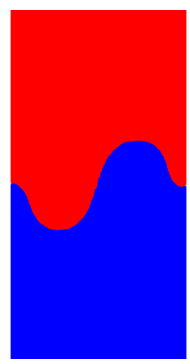

(b) $t_{*}=1.25$

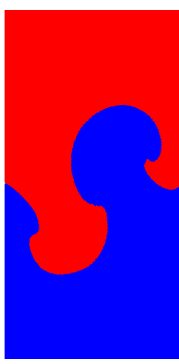

(c) $t_{*}=2.5$

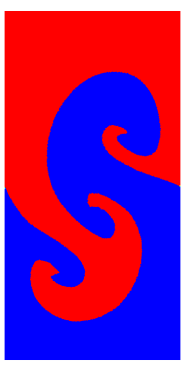

(d) $t_{*}=3.75$

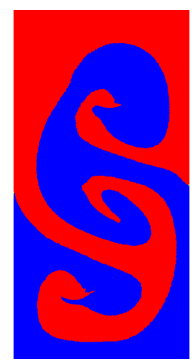

(e) $t_{*}=5$

Figure 19: Rayleigh-Taylor instability: light (blue) and heavy (red) phases evolution for a discretization of 300 particles per unit length.

in the curling up region of the mushroom-shaped heads.

Introducing a constant relative velocity (42) chosen arbitrarily with $d_{p}=0.07 \mathrm{~m}$, interfaces first diffuse and rapidly converge towards a separated state as in section 4.1. Simulations without and with relative velocity are displayed in Figure 21. One can see that without relative velocity (i.e. configuration equivalent to the multi-fluid model), phases are not fully separated as some drops remain in both phases and are under-resolved because of the size of SPH particles (a steady state is not reached even at $t_{*}=500$ even though phases are then more clearly separated). An inaccurate pressure gradient computation for those small size structures might explain this behaviour, making our mixture approach useful in such circumstances. 


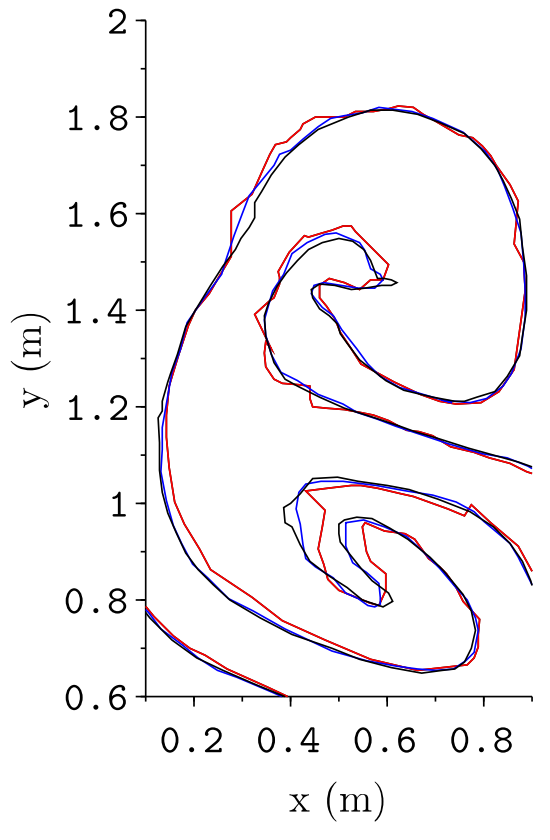

(a) Convergence study

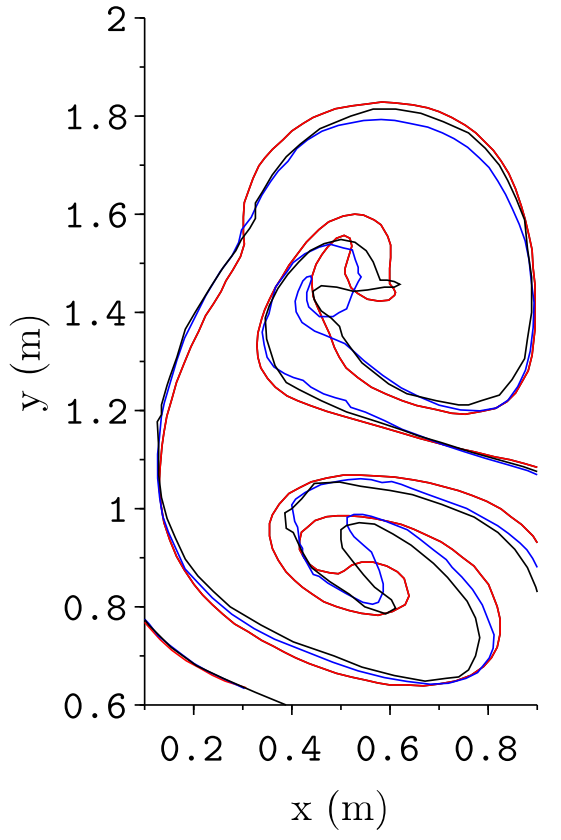

(b) Comparison with Grenier et al. (2009)

Figure 20: Comparison of the interfaces for the Rayleigh-Taylor instability case at $t_{*}=5$. On the left, a convergence study is displayed for 75 (red line), 150 (blue line) and 300 (black line) particles per unit length. On the right, a comparison is made between Level-Set (red line) and SPH (blue line) results of Grenier et al. (2009) and the present SPH model (black line) for the same resolution. 

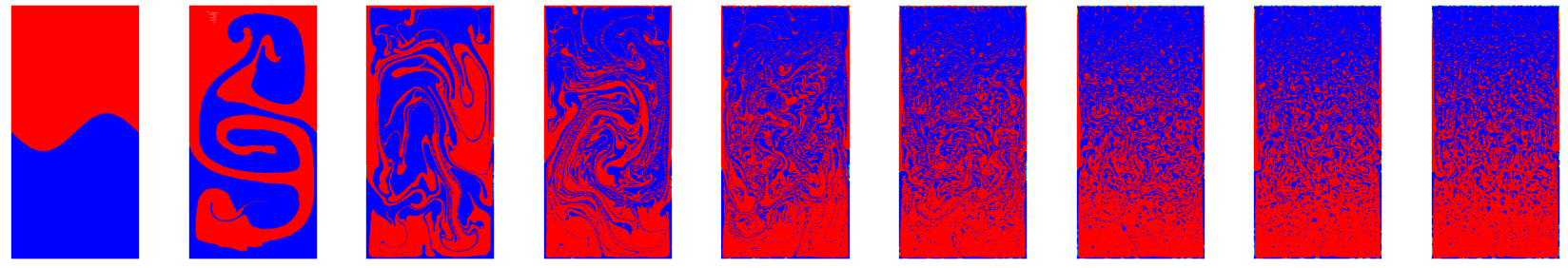

(a) $t_{*}=0$

(b) $t_{*}=6.25$

(c) $t_{*}=12.5$

(d) $t_{*}=18.75$

(e) $t_{*}=25$

(f) $t_{*}=31.25$

(g) $t_{*}=37.5(\mathrm{~h})$

(h) $t_{*}=43.75$

(i) $t_{*}=50$


(a) $t_{*}=0$

(b) $t_{*}=6.25$

(c) $t_{*}=12.5$

(d) $t_{*}=18.75$

(e) $t_{*}=25$

(f) $t_{*}=31.25$

(g) $t_{*}=37.5$

(h) $t_{*}=43.75$

(i) $t_{*}=50$

Figure 21: Rayleigh-Taylor instability: evolution of the volume fractions from the light (blue) to heavy (red) phases without (upper series) and with (lower series) relative velocity for a discretization of 300 particles per unit length.

\subsection{Limits of the mixture model: a sand dumping case}

In this section, the performances of the presented mixture model are assessed on a case of sand dumping described in (Shi et al., 2017). This case implies enriched physics compared to the previous sections, with the presence of a free surface and a physically-based relative velocity closure. $\alpha$ denotes the sediment phase, and $\beta$ the water phase. The configuration is a square water tank $(1 \mathrm{~m} \times 1 \mathrm{~m})$ with a free surface as described in Figure 22. In the middle of the domain, just under the free surface, a sand block is released initially with a volume fraction $\alpha_{0}$ equal to the maximum sediment concentration. Many different configurations were considered by (Shi et al., 2017) but we will focus on the tests for which experimental results are available. The sand cloud is initially $W_{0}=2$ or $4 \mathrm{~cm}$ wide and $H_{0}=2.5 \mathrm{~cm}$ high. The three sizes of sediment $d_{p}$ considered are 0.8 $\mathrm{mm}, 1.3 \mathrm{~mm}$ and $5 \mathrm{~mm}$ that correspond roughly to settling velocities of $12.6 \mathrm{~cm} / \mathrm{s}, 19.61 \mathrm{~cm} / \mathrm{s}$ and $49.52 \mathrm{~cm} / \mathrm{s}$, respectively. The particle discretization is $\delta r=0.005 \mathrm{~m}$.

In order to have a fair comparison with the two-fluid approach developed in (Shi et al., 2017), several changes were introduced to deal with the complete physics of this case: 


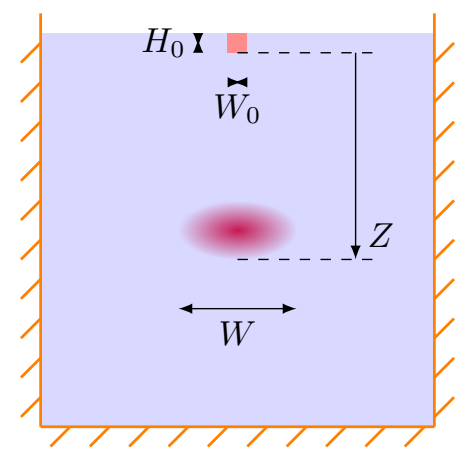

Figure 22: Sand dumping: sand cloud features.

- Computation of the sediment viscosity following Alihan and Sleath's formula Alihan \& Sleath 1987):

$$
\nu^{\alpha}=1.2 \frac{\rho^{\beta}}{\rho^{\alpha}}\left[\left(\frac{\alpha_{s m}}{\alpha}\right)^{\frac{1}{3}}-1\right]^{-2} \nu^{\beta}
$$

where $\alpha_{s m}$ is the maximum sediment volumetric concentration, that was set to 0.607 in our computations (instead of 0.606 in (Shi et al. 2017)) to avoid singularity of the viscosity at the initialization (indeed the sediment cloud starts at this concentration initially).

- Computation of a turbulent viscosity, added to the physical one, following a Smagorinsky mode 5

$$
\nu^{t}=(C \delta r)^{2}\|\boldsymbol{S}\|\left(1-\frac{\alpha}{\alpha_{s m}}\right)^{n}
$$

where $C=0.1$ and $n=5$. Please note that we are using here the mixture rate-of-strain tensor defined as:

$$
\boldsymbol{S}=\frac{1}{2}\left(\boldsymbol{\nabla} \boldsymbol{j}+{ }^{t} \boldsymbol{\nabla} \boldsymbol{j}\right) \text { and }\|\boldsymbol{S}\|=\sqrt{2 \boldsymbol{S}: \boldsymbol{S}}
$$

that differs from the phase rate-of-strain tensors used for distinct phase turbulent viscosities computations in (Shi et al., 2017). Moreover, a sensitivity analysis has been conducted in (Shi et al. 2017) to optimize the results with adequate choice of the Smagorinsky coefficients and power law, choices that might not be optimal in the framework of our mixture model.

\footnotetext{
${ }^{5}$ Some prefer using the smoothing length $h$ in place of $\delta r$. It is a matter of convenience about the definition of $C$. However, we emphasize that, following (Dehnen \& Aly [2012), $h$ is meaningless and should be replaced by the kernel standard deviation.
} 
- A relative velocity closure is deduced from (Shi et al. 2017) continuous equations. It includes the drag effect, an hindering factor computed with Richardson and Zaki formula (Richardson \& Zaki, 1997) and a turbulent diffusion of volume fraction based on a gradient transport law:

$$
\boldsymbol{v}^{r}=\frac{4}{3} \frac{\beta^{2.65}\left(\rho^{\alpha}-\rho^{\beta}\right) d_{p}}{\rho^{\beta} C_{D}\left\|\boldsymbol{v}^{r}\right\|} \frac{\nabla p}{\rho}-\frac{\nu^{t}}{S c} \frac{\nabla \alpha}{\alpha \beta}
$$

where the drag coefficient is computed according to the Reynolds number $R e=\left\|\boldsymbol{v}^{r}\right\| d_{p} / \nu^{\beta}$ following:

$$
C_{D}= \begin{cases}\frac{24}{R e}\left(1+0.15 R e^{0.687}\right) & \text { if } R e<1000 \\ 0.44 & \text { if } R e \geq 1000\end{cases}
$$

Iterations are made to converge to a well-defined value of the relative velocity, due to the implicit relation implied by the drag. The Schmidt number $S c$ is taken equal to 1 .

- Combining the momentum equations of both phases under the constant phase density hypothesis, one can obtain a momentum equation for the mixture, distinct from (9). The pressure gradient term is modified and a convective transfer term appears. The additional viscous terms are neglected, as they trigger some unphysical behaviours at the beginning of the simulation and do not change the results described in this paper. Hence equation (9) was changed into:

$$
\frac{d \boldsymbol{j}}{d t}=-\left(\frac{\alpha}{\rho^{\alpha}}+\frac{\beta}{\rho^{\beta}}\right) \boldsymbol{\nabla} p+\frac{1}{\rho} \boldsymbol{\nabla} \cdot \boldsymbol{T}+\boldsymbol{g}-\boldsymbol{\nabla} \cdot\left(\alpha \beta \boldsymbol{v}^{r} \otimes \boldsymbol{v}^{r}\right)
$$

A different state equation is used in (Shi et al., 2017) but the tests made did not highlight any significant modification of the following results. One can follow temporally the evolution of the cloud, defined as the particles with a sediment volume fraction $\alpha$ equal to at least $5 \%$ of the maximum value in the domain at the considered instant, through its vertical position $Z$, its width $W$ and its frontal velocity $w_{c}$. Moreoever, one can follow the free surface fluctuations at the release point $z_{w}$ : this must be handled with care however, as the flucutations after the initial jump are of the order of the particle size. The adimensionalization coefficients used in the graphs are $L_{0}=\sqrt{W_{0} H_{0}}$ and $u_{0}=\sqrt{\left(\alpha_{0} \rho^{\alpha}+\left(1-\alpha_{0}\right) \rho^{\beta}\right) g L_{0} / \rho^{\beta}}$.

Numerical results of the mixture model are compared with SPH results of (Shi et al., 2017) and experimental results of (Nakasuji et al. 1990) for the frontal velocity of the cloud in Figures 23 and 24 and for the cloud width in Figures 25 and 26 . The frontal velocity is overestimated in the late time of the simulation, once the initial transient is passed, even though there is an overall 


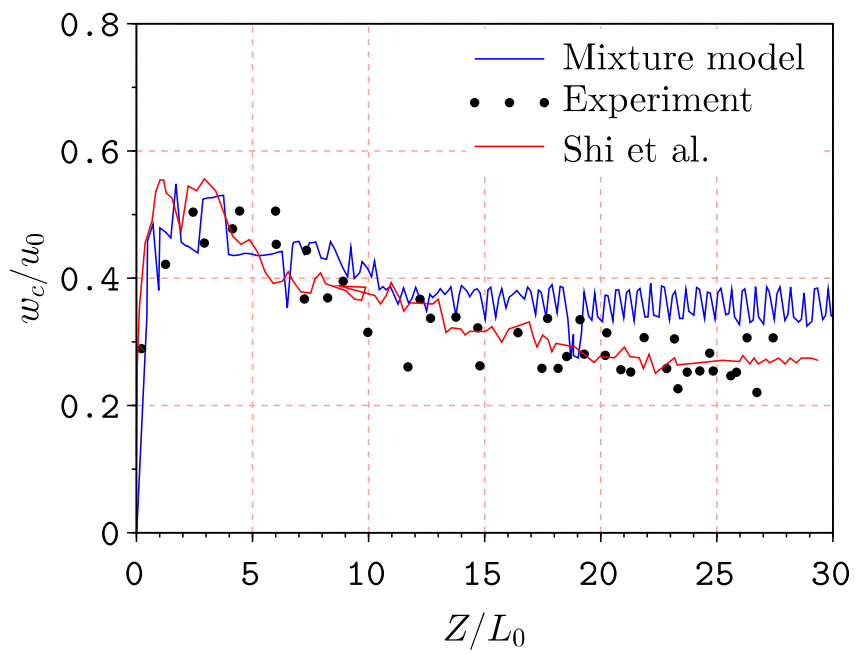

Figure 23: Comparison of the cloud frontal velocity for the mixture model, (Shi et al., 2017) numerical results and Nakasuji et al. 1990) experimental results for $d_{p}=0.8 \mathrm{~mm}$ and $W_{0}=2 \mathrm{~cm}$.

good trend. The cloud falls more rapidly than in (Shi et al., 2017), especially for large sediments (i.e. large relative velocities). The cloud width is correctly reproduced for small sediments in the first moments of the fall, until $Z=4 L_{0}$, then the model fails at reproducing the change of slope with an increase of the cloud width growth. Figure 27 displays the comparison of the free surface fluctuations: they are well reproduced. Cloud repartition is displayed in Figure 28. The two-phase model of (Shi et al. 2017) predicts a double-peak turbidity distribution (two symmetrical cores) of the sediment cloud (no experimental evidence found). The mixture model fails at reproducing this topology: only one turbidity core is detected, at least at low resolution. With a discretization twice finer, a double-peak configuration appears as highlighted in Figure 29. According to (Nguyen et al. 2012), vorticity is largely underestimated with a single-phase model: the absence of recirculation within the lower part of the cloud prevents any separation of the cloud. It can also explain that the cloud falls more rapidly with the mixture model, without any recirculation slowing down the progression of the cloud.

\section{Conclusion}

In this paper, a two-phase SPH mixture model suited to high density ratios was introduced. Thanks to a finite volume-like reasoning, a realizable numerical scheme, conservative with respect 


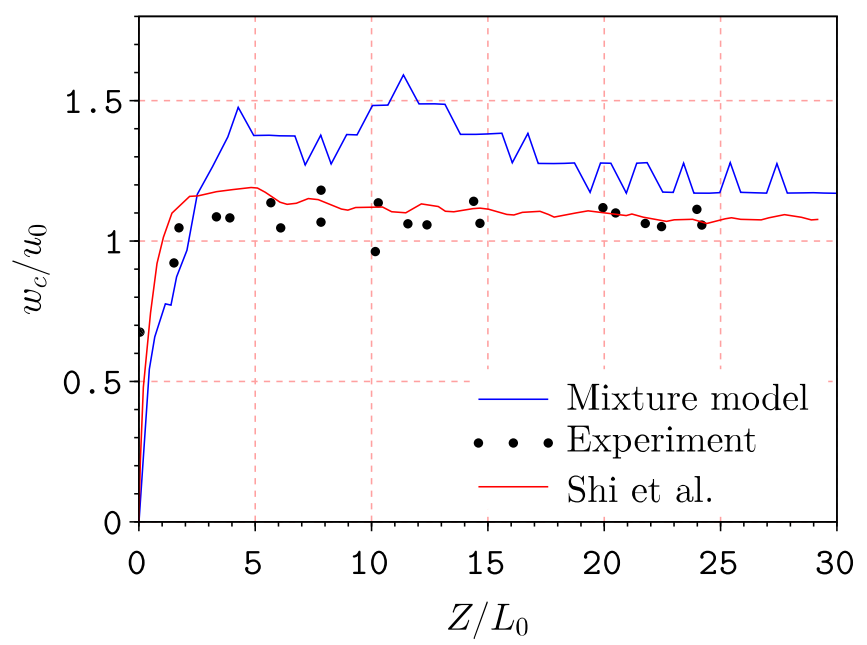

Figure 24: Comparison of the cloud frontal velocity for the mixture model, (Shi et al., 2017) numerical results and Nakasuji et al. 1990) experimental results for $d_{p}=5 \mathrm{~mm}$ and $W_{0}=2 \mathrm{~cm}$.

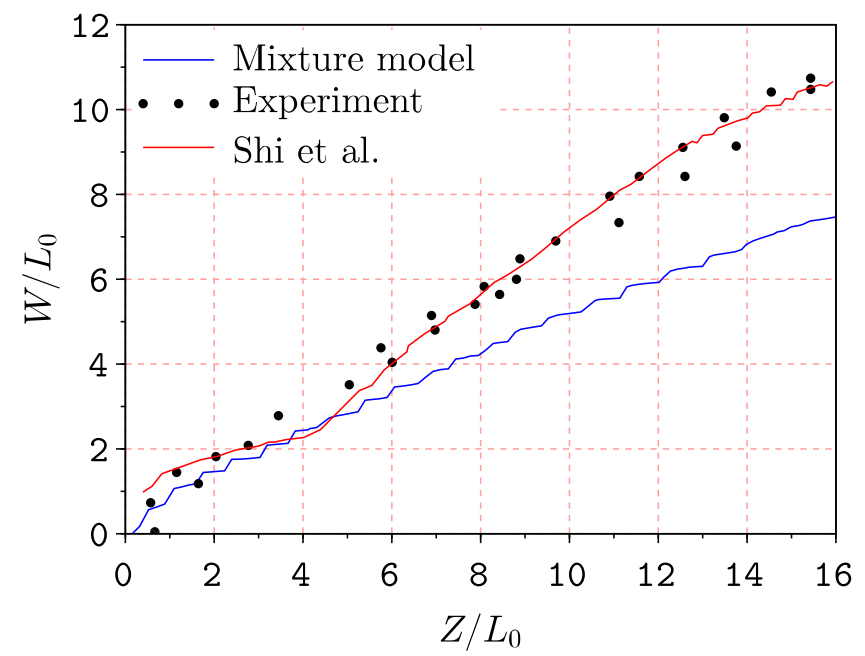

Figure 25: Comparison of the cloud width for the mixture model, (Shi et al., 2017) numerical results and (Nakasuji et al. 1990) experimental results for $d_{p}=1.3 \mathrm{~mm}$ and $W_{0}=4 \mathrm{~cm}$. 


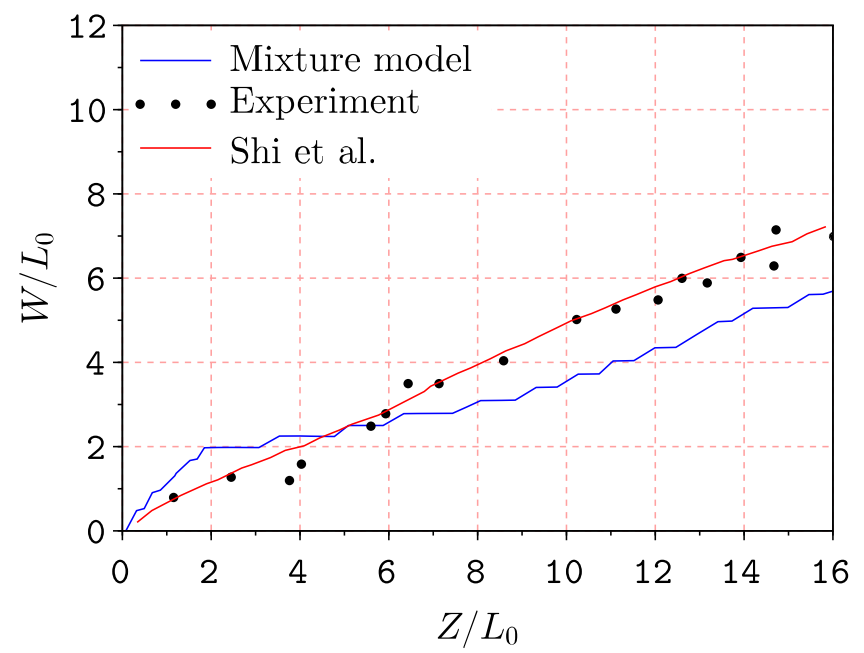

Figure 26: Comparison of the cloud width for the mixture model, (Shi et al. 2017) numerical results and (Nakasuji et al. 1990) experimental results for $d_{p}=5 \mathrm{~mm}$ and $W_{0}=4 \mathrm{~cm}$.

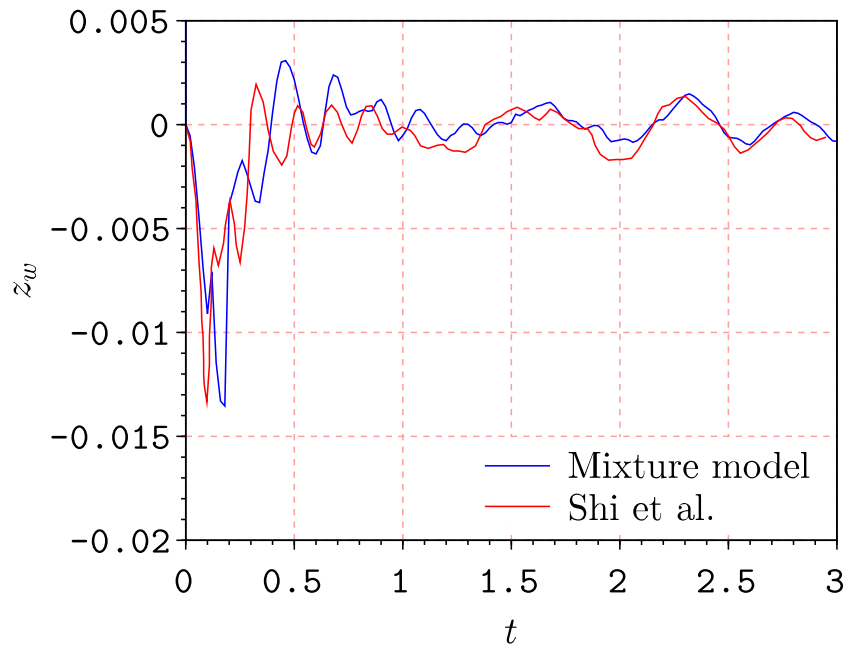

Figure 27: Comparison of the fluctuations of the free surface for the mixture model and (Shi et al. 2017) numerical results for $d_{p}=0.8 \mathrm{~mm}$ and $W_{0}=2 \mathrm{~cm}$. 


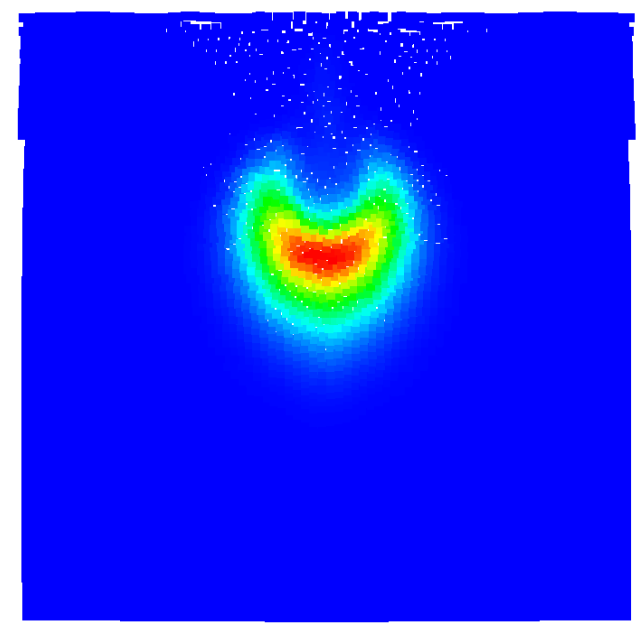

(a) Mixture model

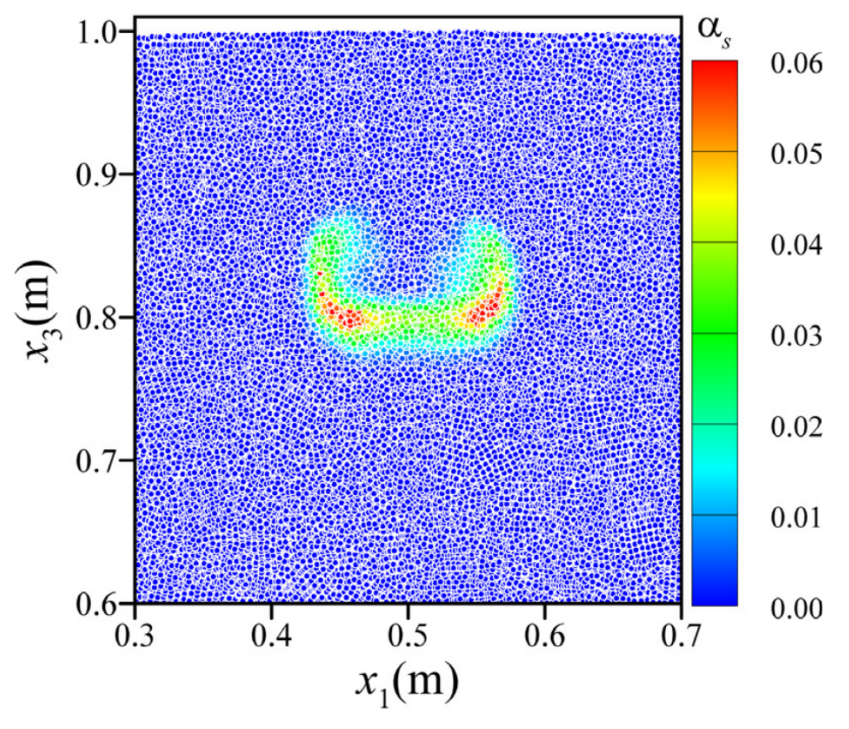

(b) (Shi et al. 2017)

Figure 28: Comparison of the sediment clouds at $t=1 \mathrm{~s}$ for $d_{p}=0.8 \mathrm{~mm}$ and $W_{0}=2 \mathrm{~cm}$.

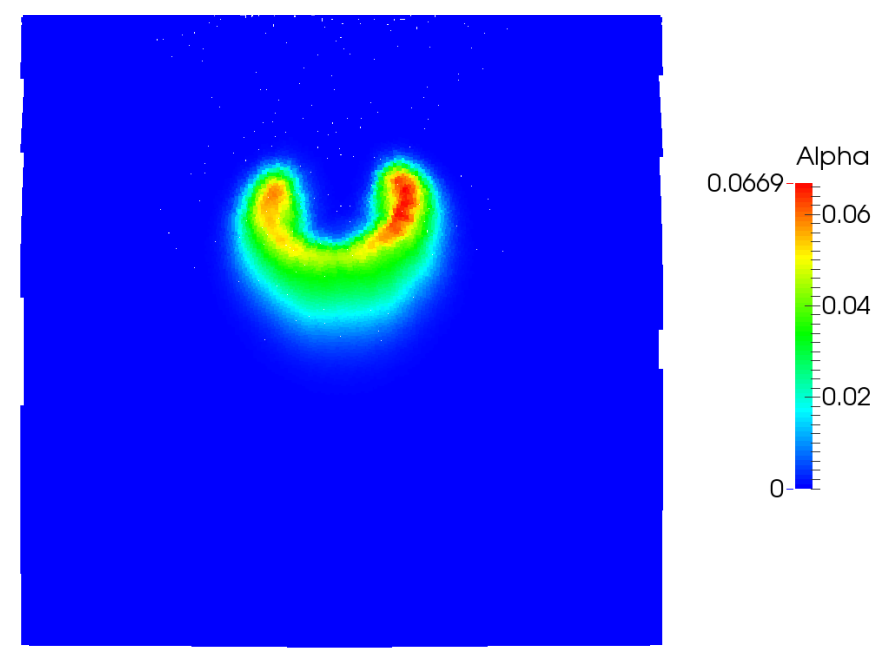

Figure 29: Sediment cloud for the finest resolution $\delta r=0.0025 \mathrm{~m}, d_{p}=0.8 \mathrm{~mm}$ and $W_{0}=2 \mathrm{~cm}$. 
to the relative velocity contribution, has been derived. It proved to give good results for several test cases. Separation cases were proposed to check that conservation and realizability were achieved, while the accuracy of the model was investigated with a two-phase mixture Poiseuille flow. A Rayleigh-Taylor instability case was also tested. The results are overall satisfactory. Limits of the model have been highlighted by a sand dumping case for which the model failed at reproducing the correct falling velocity and topology of a sand cloud at a given discretization compared to a two-velocity approach. The pressure gradient computation for high density ratios needs more investigation: indeed, the multi-fluid operator did not appear sufficient to prevent numerical instabilities close to the interface in the separation cases. A momentum equation consistent with the volumebased formulation shall also be further investigated. Second order schemes using slope limiters can be studied to improve the convergence order.

\section{Appendix A. Volume diffusion}

We follow the derivation of Brezzi \& J. Pitkäranta (1984), but apply it to the volume equation (18) instead of the density equation. Using the momentum equation (9), the discrete volume equation writes:

$$
\begin{aligned}
\frac{V_{a}^{n+1}-V_{a}^{n}}{\delta t}= & V_{a}^{n} D_{a}\left\{\boldsymbol{j}_{b}^{n+1}\right\} \\
\approx & V_{a}^{n} D_{a}\left\{\boldsymbol{j}_{b}^{n}-\frac{\delta t}{\rho_{a}^{n}} \boldsymbol{G}_{a}\left\{p_{b}^{n}\right\}+\frac{\delta t}{\rho_{a}^{n}} \rho_{a}^{n} \boldsymbol{g}\right\} \\
\approx & V_{a}^{n} D_{a}\left\{\boldsymbol{j}_{b}^{n}\right\} \\
& -V_{a}^{n} D_{a}\left\{\frac{\delta t}{\rho_{a}^{n}} \boldsymbol{G}_{a}\left\{p_{b}^{n}\right\}-\delta t \boldsymbol{G}_{a}\left\{\boldsymbol{g} \cdot \boldsymbol{r}_{b}^{n}\right\}\right\}
\end{aligned}
$$

where $D_{a}$ and $\boldsymbol{G}_{a}$ stands for the discrete divergence and gradient operators and $\delta t$ the time step. Using the Laplacian operator $L_{a}$, which is slightly different from the divergence of a gradient in $\mathrm{SPH}$, the volume diffusion term $\mathcal{F}_{a}$ writes:

$$
\mathcal{F}_{a}=V_{a} L_{a}\left\{\frac{\delta t}{\rho_{b}}, p_{b}\right\}-V_{a} L_{a}\left\{\delta t, \boldsymbol{g} \cdot \boldsymbol{r}_{b}\right\}
$$

It is written discretely as

$$
\mathcal{F}_{a}^{n}=2 V_{a} \delta t \sum_{b} V_{b} \frac{1}{r_{a b}}\left(\frac{2}{\rho_{a}+\rho_{b}}\left(p_{a}-p_{b}\right)-\boldsymbol{g} \cdot \boldsymbol{r}_{a b}\right) \nabla w_{a b}
$$


The easiest approach is now to introduce the usual volume diffusion in both $V^{\alpha}$ and $V^{\beta}$ equations, but weighted respectively by $\alpha$ and $\beta$. The new phase volumes computed by (33) and (34) being denoted $V^{\alpha, *}, V^{\beta, *}$, using a volume diffusion coefficient $\Lambda$ (generally $\Lambda=0.1$ ), we finally write at time $n+1$ :

$$
\begin{aligned}
V_{a}^{\alpha, n+1} & =V_{a}^{\alpha, *}+\frac{\delta t}{\gamma_{a}} \Lambda \alpha_{a}^{n} \mathcal{F}_{a}^{n} \\
V_{a}^{\beta, n+1} & =V_{a}^{\beta, *}+\frac{\delta t}{\gamma_{a}} \Lambda \beta_{a}^{n} \mathcal{F}_{a}^{n}
\end{aligned}
$$

\section{Appendix B. Derivation of a condition for positiveness of phase volumes}

In the following, the particle $a$ considered is far from boundaries (no boundary term) and we neglect the volume diffusion term. We search for a sufficient condition of realizability of the phase volume using equation 49 :

$$
\begin{aligned}
& \frac{d V_{a}^{\alpha}}{d t}=\alpha_{a} \frac{d V_{a}}{d t}-\sum_{b}\left[\alpha_{a} \beta_{b}\left[\boldsymbol{v}_{0, a \mid b} \cdot \boldsymbol{S}_{a \mid b}\right]^{+}+\alpha_{b} \beta_{a}\left[\boldsymbol{v}_{0, a \mid b} \cdot \boldsymbol{S}_{a \mid b}\right]^{-}\right] \\
& +K \sum_{b} \frac{\beta_{a}+\beta_{b}}{2} \frac{\alpha_{a}-\alpha_{b}}{r_{a b}} \frac{\boldsymbol{r}_{a b} \cdot \boldsymbol{S}_{a \mid b}}{r_{a b}}
\end{aligned}
$$

Let us note

$$
\phi(r)=-2 \frac{\boldsymbol{r} \cdot \boldsymbol{\nabla} w}{r^{2}} \text { so that } \phi_{a b}=-2 \frac{\boldsymbol{r}_{a b} \cdot \boldsymbol{\nabla} w_{a b}}{r_{a b}^{2}} \geq 0
$$

Replacing $\alpha, \beta$ and $\boldsymbol{S}_{a \mid b}$ by their definitions:

$$
\begin{aligned}
& \frac{d V_{a}^{\alpha}}{d t}=\frac{V_{a}^{\alpha}}{V_{a}} \frac{d V_{a}}{d t}-2 \sum_{b}\left[V_{a}^{\alpha} V_{b}^{\beta}\left[\boldsymbol{v}_{0, a \mid b} \cdot \nabla w_{a b}\right]^{+}+V_{b}^{\alpha} V_{a}^{\beta}\left[\boldsymbol{v}_{0, a \mid b} \cdot \nabla w_{a b}\right]^{-}\right] \\
& -\frac{1}{2} K \sum_{b}\left(V_{b} V_{a}^{\beta}+V_{a} V_{b}^{\beta}\right)\left(\frac{V_{a}^{\alpha}}{V_{a}}-\frac{V_{b}^{\alpha}}{V_{b}}\right) \phi_{a b}
\end{aligned}
$$

Using $V^{\beta}=V-V^{\alpha}$ :

$$
\begin{aligned}
& \frac{d V_{a}^{\alpha}}{d t}=\frac{V_{a}^{\alpha}}{V_{a}} \frac{d V_{a}}{d t}-2 \sum_{b}\left[V_{a}^{\alpha} V_{b}^{\beta}\left[\boldsymbol{v}_{0, a \mid b} \cdot \nabla w_{a b}\right]^{+}+V_{b}^{\alpha} V_{a}^{\beta}\left[\boldsymbol{v}_{0, a \mid b} \cdot \nabla w_{a b}\right]^{-}\right] \\
& -K \sum_{b}\left(\left(1-\frac{\alpha_{a}}{2}\right) V_{b} V_{a}^{\alpha}-\left(1-\frac{\alpha_{b}}{2}\right) V_{a} V_{b}^{\alpha}\right) \phi_{a b}
\end{aligned}
$$


Discretizing temporally $\left(V_{a}^{n+1}\right.$ is taken as an input here, computed separately before, its factor is explicit):

$$
\begin{aligned}
& \frac{V_{a}^{\alpha, n+1}-V_{a}^{\alpha, n}}{\delta t}=\frac{V_{a}^{\alpha, n}}{V_{a}^{n}} \frac{V_{a}^{n+1}-V_{a}^{n}}{\delta t} \\
& -2 \sum_{b}\left[V_{a}^{\alpha, n} V_{b}^{\beta, n}\left[\boldsymbol{v}_{0, a \mid b} \cdot \nabla w_{a b}^{n}\right]^{+}+V_{b}^{\alpha, n} V_{a}^{\beta, n}\left[\boldsymbol{v}_{0, a \mid b} \cdot \nabla w_{a b}^{n}\right]^{-}\right] \\
& -K \sum_{b}\left(\left(1-\frac{\alpha_{a}^{n}}{2}\right) V_{b}^{n} V_{a}^{\alpha, n}-\left(1-\frac{\alpha_{b}^{n}}{2}\right) V_{a}^{n} V_{b}^{\alpha, n}\right) \phi_{a b}^{n}
\end{aligned}
$$

Rearranging and using $[x, 0]^{-}=-[-x, 0]^{+}$, we finally get:

$$
\begin{aligned}
& V_{a}^{\alpha, n+1}=V_{a}^{\alpha, n}\left[\frac{V_{a}^{n+1}}{V_{a}^{n}}-\delta t \sum_{b} V_{b}^{n}\left(\beta_{b}^{n}\left[2 \boldsymbol{v}_{0, a \mid b} \cdot \nabla w_{a b}^{n}\right]^{+}+K\left(1-\frac{\alpha_{a}^{n}}{2}\right) \phi_{a b}^{n}\right)\right] \\
& +\delta t \sum_{b} V_{b}^{\alpha, n}\left(V_{a}^{\beta, n}\left[-2 \boldsymbol{v}_{0, a \mid b} \cdot \nabla w_{a b}^{n}\right]^{+}+K\left(1-\frac{\alpha_{b}^{n}}{2}\right) V_{a}^{n} \phi_{a b}^{n}\right)
\end{aligned}
$$

If we assume $V^{\alpha, n}>0$ and $V^{\beta, n}>0$ for every particle (therefore $V^{n}>0$ ), then we have $V^{\alpha, n+1}>0$ (and symmetrically $V^{\beta, n+1}>0$ ) under the condition that:

$$
\delta t \sum_{b} V_{b}^{n}\left(\beta_{b}^{n}\left[2 \boldsymbol{v}_{0, a \mid b} \cdot \nabla w_{a b}^{n}\right]^{+}+K\left(1-\frac{\alpha_{a}^{n}}{2}\right) \phi_{a b}^{n}\right) \leq \frac{V_{a}^{n+1}}{V_{a}^{n}}
$$

as the second term of the left hand side of equation (B.6) is always positive.

We now search for an upper bound of the left hand side term of the unequality (B.7). One can first write:

$$
\delta t \sum_{b} V_{b}^{n}\left(\left[2 \boldsymbol{v}_{0, a \mid b} \cdot \nabla w_{a b}^{n}\right]^{+}+K \phi_{a b}^{n}\right) \leq \frac{V_{a}^{n+1}}{V_{a}^{n}}
$$

In the continuous interpolation framework, we have according to Violeau \& Leroy (2014):

$$
\int_{\Omega} \phi(r) d \boldsymbol{r} \sim \frac{2}{\sigma^{2}}
$$

with the kernel standard deviation $\sigma=\sqrt{\frac{5}{18}} h$ for the $\mathcal{C}^{2}$ Wendland kernel in 2 dimensions.

Noting $v_{\max }=\max \left(\left|\boldsymbol{v}_{0}\right|\right)$, an upper bound can be written:

$$
\delta t\left(\eta \frac{v_{\max }}{\sigma}+\frac{K}{\sigma^{2}}\right) \leq \xi \frac{V_{a}^{n+1}}{V_{a}^{n}}
$$

where $\eta$ and $\xi$ are unknown. Volumes do not vary a lot so that we may consider that the ratio $V_{a}^{n+1} / V_{a}^{n}$ is close to 1 . Noting $P e_{\sigma}$ the Péclet number $\frac{v_{\max } \sigma}{K}$, one can deduce an approximate upper bound to the CFL coefficient:

$$
C F L=\frac{v_{\max } \delta t}{\sigma} \leq \xi\left(\eta+\frac{1}{P e_{\sigma}}\right)^{-1}
$$




\section{Appendix C. Analytical solution of the two-phase mixture Poiseuille flow}

At steady state, the system of equations to solve (8) and (9) becomes:

$$
\begin{gathered}
\boldsymbol{\nabla} \cdot\left(\alpha \beta \boldsymbol{v}^{r}\right)=0 \\
-\frac{1}{\rho} \nabla p+\frac{1}{\rho} \boldsymbol{\nabla} \cdot\left(\rho \nu\left(\boldsymbol{\nabla} \boldsymbol{j}+{ }^{t} \boldsymbol{\nabla} \boldsymbol{j}\right)\right)+\boldsymbol{g}=\mathbf{0}
\end{gathered}
$$

Under the longitudinal periodicity condition and projecting the momentum equation along the longitudinal axis, this system becomes:

$$
\begin{gathered}
\frac{d}{d z}\left(\alpha \beta \boldsymbol{v}^{r} \cdot \boldsymbol{e}_{z}\right)=0 \\
\frac{d}{d z}\left(\rho \nu \frac{d}{d z} \boldsymbol{j} \cdot \boldsymbol{e}_{z}\right)=0
\end{gathered}
$$

No-flux condition at boundaries implies that at steady state equation C.3 becomes after integration:

$$
\boldsymbol{v}^{r} \cdot \boldsymbol{e}_{z}=\boldsymbol{v}_{0} \cdot \boldsymbol{e}_{z}-K \frac{d \alpha}{d z}=0
$$

In the simplified momentum equation C.4 , $\rho=\alpha \rho^{\alpha}+\beta \rho^{\beta}$ and $\nu=\alpha \nu^{\alpha}+\beta \nu^{\beta}$ are variable and depend on the volume fraction solution of equation C.5. The adimensionalized solution for a constant kinematic viscosity $\nu=\nu^{\alpha}=\nu^{\beta}$ writes:

- Volume fraction

$$
\alpha\left(z_{*}\right)=\alpha_{1} \exp \left(P e z_{*}\right)
$$

- Longitudinal velocity

$$
\begin{aligned}
& j_{*}\left(z_{*}\right)=\frac{3}{2}\left(1-z_{*}^{2}\right) \\
& +\frac{3}{P e^{2}}\left[L i_{2, r}\left(z_{*}\right)+P e z_{*} \ln _{r}\left(z_{*}\right)+C_{1}\left(P e^{2} z_{*}+P e \ln _{r}\left(z_{*}\right)\right)+C_{2}\right]
\end{aligned}
$$

where $z_{*}=z / e, j_{*}=\boldsymbol{j} \cdot \boldsymbol{e}_{x} / U$ with $U=\rho^{\beta} F e^{2} /\left(3 \mu^{\beta}\right)$ (discharge for the usual single-fluid Poiseuille flow), $r=\left(\rho^{\alpha}-\rho^{\beta}\right) / \rho^{\beta}$ and $P e=e\left|\boldsymbol{v}_{0}\right| / K$ the Péclet number. We used the notations $\ln _{r}\left(z_{*}\right)=\ln \left(1+\alpha_{1} r \exp \left(P e z_{*}\right)\right)$ and $L i_{2, r}\left(z_{*}\right)=L i_{2}\left(-\alpha_{1} r \exp \left(P e z_{*}\right)\right)$ where $L i_{2}$ is the 
dilogarithm function ${ }^{6} C_{1}$ and $C_{2}$ are deduced from the no-slip condition at walls:

$$
\begin{array}{r}
C_{1}=\frac{-L i_{2, r}(1)+L i_{2, r}(-1)-P e\left(\ln _{r}(1)+\ln _{r}(-1)\right)}{2 P e^{2}+P e\left(\ln _{r}(1)-\ln _{r}(-1)\right)} \\
C_{2}=\frac{\left[P e \ln _{r}(1)+L i_{2, r}(1)\right]\left[\ln _{r}(-1)-P e\right]}{2 P e+\ln _{r}(1)-\ln _{r}(-1)} \\
+\frac{\left[P e \ln _{r}(-1)-L i_{2, r}(-1)\right]\left[\ln _{r}(1)+P e\right]}{2 P e+\ln _{r}(1)-\ln _{r}(-1)}
\end{array}
$$

$\alpha_{1}$ is computed thanks to the conservation of volume:

$$
\frac{\alpha_{1}}{\alpha_{0}}=\frac{P e}{\sinh (P e)}
$$

To avoid complete separation of phases, (C.6) gives a condition on $\alpha_{1}$ :

$$
0 \leq \alpha_{1} \leq \exp (-P e)
$$

And therefore a condition on the initial uniform volume fraction $\alpha_{0}$ using [C.10]:

$$
0 \leq \alpha_{0} \leq \frac{1-\exp (-2 P e)}{2 P e}
$$

As a second step, one can consider a case with a varying mixture kinematic viscosity. Noting $R=\left(\nu^{\alpha}-\nu^{\beta}\right) / \nu^{\beta}$, only the longitudinal velocity profile is modified:

$$
\begin{aligned}
j_{*}\left(z_{*}\right)= & \frac{3}{2}\left(1-z_{*}^{2}\right)+\frac{3}{(R-r) P e^{2}}\left[P e\left(R \ln _{R}\left(z_{*}\right)-r \ln _{r}\left(z_{*}\right)\right) z_{*}\right. \\
& +R L i_{2, R}\left(z_{*}\right)-r L i_{2, r}\left(z_{*}\right)-r \ln _{R}\left(z_{*}\right)+r \ln _{r}\left(z_{*}\right) \\
& \left.+C_{1}\left((R-r) P e^{2} z_{*}-P e\left(R \ln _{R}\left(z_{*}\right)+r \ln _{r}\left(z_{*}\right)\right)\right)+C_{2}\right]
\end{aligned}
$$

where the additional notations $\ln _{R}\left(z_{*}\right)=\ln \left(1+\alpha_{1} R \exp \left(P e z_{*}\right)\right)$ and $L i_{2, R}\left(z_{*}\right)=L i_{2}\left(-\alpha_{1} R \exp \left(P e z_{*}\right)\right)$ are used. $C_{1}$ and $C_{2}$ are deduced from the no-slip condition at walls:

$$
\begin{aligned}
& C_{1}=\frac{-(r+P e R) \ln _{R}(-1)+r(P e+1) \ln _{r}(-1)}{2(R-r) P e^{2}-P e\left(R\left(\ln _{R}(1)-\ln _{R}(-1)\right)+r\left(\ln _{r}(1)-\ln _{r}(-1)\right)\right)} \\
& +\frac{-R\left(L i_{2, R}(1)-L i_{2, R}(-1)\right)+r\left(L i_{2, r}(1)-L i_{2, r}(-1)\right)}{2(R-r) P e^{2}-P e\left(R\left(\ln _{R}(1)-\ln _{R}(-1)\right)+r\left(\ln _{r}(1)-\ln _{r}(-1)\right)\right)} \\
& +\frac{(r-P e R) \ln _{R}(1)+r(P e-1) \ln _{r}(1)}{2(R-r) P e^{2}-P e\left(R\left(\ln _{R}(1)-\ln _{R}(-1)\right)+r\left(\ln _{r}(1)-\ln _{r}(-1)\right)\right)}
\end{aligned}
$$

\footnotetext{
${ }^{6} L i_{2}(x)=-\int_{0}^{x} \frac{\ln (1-t)}{t} d t$. If $|x| \leq 1$, the series expression writes $L i_{2}(x)=\sum_{n} \frac{x^{n}}{n^{2}}$
} 


$$
\begin{aligned}
& C_{2}=-C_{1}\left[(R-r) P e^{2}-P e\left(R \ln _{R}(1)+r \ln _{r}(1)\right)\right] \\
& -P e\left[R \ln _{R}(1)-r \ln _{r}(1)\right]-R L i_{2, R}(1)+r L i_{2, r}(1)+r \ln _{R}(1)-r \ln _{r}(1)
\end{aligned}
$$

\section{Acknowledgment}

This work was funded by EDF R\&D and the French Research Agency (CIFRE grant agreement \#2016-0362).

\section{References}

Alihan, R., \& Sleath, J. F. (1987). Sediment Transport in Oscillatory Flow Over Flat Beds. Journal of Hydraulic Engineering, 113, 308-321. doi 10.1061/(ASCE) 0733-9429(1987)113:3(308).

Baer, M. R., \& Nunziato, J. W. (1986). A Two-phase Mixture Theory for the Deflagration-toDetonation Transition (DDT) in Reactive Granular Materials. Journal of Multiphase flows, 12, 861-889. doi $10.1016 / 0301-9322(86) 90033-9$.

Brethour, J., \& Hirt, C. W. (2009). Drift model for two-component flows. Technical Report Flow Science, Inc. Technical note FSI-09-TN83Rev.

Brezzi, F., \& J. Pitkäranta (1984). On the stabilization of finite element approximations of the Stokes equations. Efficient Solutions of Elliptic Systems, Notes on Numerical Fluid Mechanics, 10, 11-19. doi $10.1007 / 978-3-663-14169-3 \_2$.

Chanteperdrix, G. (2004). Modélisation et simulation numérique d'écoulements diphasiques à interface libre. Application à l'étude des mouvements de liquides dans les réservoirs de véhicules spatiaux. Ph.D. thesis Centre National d'Études Spatiales. 39-42.

Colagrossi, A., \& Landrini, M. (2003). Numerical simulation of interfacial flows by smoothed particle hydrodynamics. Journal of Computational Physics, 191, 448-475. doi 10.1016/S0021-9991(03) 00324-3.

Cole, R. H. (1948). Underwater explosions. chapter 2. Princeton University Press. 
Cueille, P. V. (2005). Modélisation par Smoothed Particle Hydrodynamic des phénomènes de diffusion présents dans un écoulement. Ph.D. thesis INSA de Toulouse.

Dehnen, W., \& Aly, H. (2012). Improving convergence in smoothed particle hydrodynamics simulations without pairing instability. Monthly Notices of the Royal Astronomical Society, 425, 1068-1082. doi:10.1111/j.1365-2966.2012.21439.x.

Español, P., \& Revenga, M. (2003). Smoothed dissipative particle dynamics. Physical Review E, 67. doi $10.1103 /$ PhysRevE.67.026705

Ferrand, M., Joly, A., Kassiotis, C., Violeau, D., Leroy, A., Morel, F.-X., \& Rogers, B. D. (2017). Unsteady open boundaries for SPH using semi-analytical conditions and Riemann solver in 2D. Computer Physics Communications, 210, 29-44. doi:10.1016/j.cpc.2016.09.009.

Freitas, C. J. (1995). Perspective: Selected Benchmarks from Commercial CFD Codes. Transactions of the ASME, 117, 208-218. doi $10.1115 / 1.2817132$.

Gallouët, T., Hérard, J.-M., \& Seguin, N. (2004). Numerical modeling of two-phase flows using the two-fluid two-pressure approach. Mathematical Models and Methods in Applied Sciences, 14, 663-700. doi:10.1142/S0218202504003404.

Ghaitanellis, A., Violeau, D., Leroy, A., Joly, A., \& Ferrand, M. (2015). Application of the unified semi-analytical wall boundary conditions to multi-phase SPH. Proceedings of 10th international SPHERIC workshop, Parma, Italy.

Gong, K., Shao, S., Liu, H., Wang, B., \& Tan, S.-K. (2016). Two-phase SPH Simulation of Fluid-Structure Interactions. Journal of Fluids and Structures, 65, 155-179. doi $10.1016 / \mathrm{j}$. jfluidstructs.2016.05.012.

Grenier, N. (2009). Modélisation numérique par la méthode SPH de la séparation eau-huile dans les séparateurs gravitaires. Ph.D. thesis Ecole Centrale de Nantes, France.

Grenier, N., Antuono, M., Colagrossi, A., Touzé, D. L., \& Alessandrini, B. (2009). An Hamiltonian interface SPH formulation for multi-fluid and free surface flows. Journal of Computational Physics, 228, 8380-8393. doi 10.1016/j.jcp.2009.08.009. 
Hirt, C. W., \& Nichols, B. D. (1981). Volume of Fluid (VOF) method for the dynamics of free boundaries. Journal of Computational Physics, 39, 201-225. doi:10.1016/0021-9991(81)90145-5.

Ishii, M., \& Hibiki, T. (2011). Thermo-fluid dynamics of two-phase flow - Second Edition. chapter 5,13. Springer.

Jeelani, S. A. K., \& Hartland, S. (1998). Effect of dispersion properties on the separation of batch liquid-liquid dispersions. Industrial \& Engineering Chemistry Research, 37, 547554. doi:10.1021/ ie970545a.

Kwon, J., \& Monaghan, J. J. (2015). Sedimentation in homogeneous and inhomogeneous fluids using SPH. International Journal of Multiphase Flow, 72, 155-164. doi 10.1016/j. ijmultiphaseflow.2015.02.004.

Labois, M. (2008). Modélisation des déséquilibres mécaniques dans les écoulements diphasiques : approches par relaxation et par modèle réduit. Ph.D. thesis Université de Provence. 24-27.

Larreteguy, A. E., Barceló, L. F., \& Caron, P. A. (2017). A bounded upwind-downwind semidiscrete scheme for finite volume methods for phase separation problems. Applied Mathematical Modelling, 50, 118-134. doi $10.1016 /$ j.apm.2017.05.003.

Manninen, M., \& Taivassalo, V. (1996). On the mixture model for multiphase flow. Valtion Teknillinen Tutkimuskeskus, 288.

Marrone, S., Antuono, M., Colagrossi, A., \& Graziani, G. (2011). $\delta$-SPH model for simulating violent impact flows. Computer Methods in Applied Mechanics and Engineering, 200, 15261542. doi $10.1016 / \mathrm{j} \cdot \mathrm{cma} \cdot 2010.12 .016$

Mayrhofer, A., Rogers, B. D., Violeau, D., \& Ferrand, M. (2013). Investigation of wall bounded flows using SPH and the unified semi-analytical wall boundary conditions. Computer Physics Communications, 184, 2515-2527. doi j.cpc.2013.07.004.

Monaghan, J. J. (1994). Simulating free surface flows with SPH. Journal of Computational Physics, 110, 399-406. doi $10.1006 / \mathrm{jcph} .1994 .1034$

Monaghan, J. J. (1997). Implicit SPH drag and dusty gas dynamics. Journal of Computational Physics, 138, 801-820. doi:10.1006/jcph.1997.5846 
Monaghan, J. J., \& Kocharyan, A. (1995). SPH simulation of multi-phase flow. Computer Physics Communications, 87, 225-235. doi 10.1016/0010-4655(94)00174-Z.

Monaghan, J. J., \& Rafiee, A. (2012). A simple SPH algorithm for multi-fluid flow with high density ratios. International Journal for Numerical Methods in Fluids, 71, 537-561. doi:10.1002/fld. 3671 .

Morris, J. P., Fow, P. J., \& Zhu, Y. (1997). Modeling low Reynolds number incompressible flows using SPH. Journal of Computational Physics, 136, 214-226. doi 10.1006/jcph.1997.5776.

Nakasuji, K., Tamai, M., \& Murota, A. (1990). Dynamic behaviour of sand clouds in water. Proceedings of the International conference on Physics: Modeling of Transport and Dispersion, Massachusetts Institute of Technology, Boston, United States.

Neuhauser, M. (2015). Development of a coupled SPH-ALE/Finite Volume method for the simulation of transient flows in hydraulic machines. Ph.D. thesis.

Nguyen, D. H., Levy, F., Bang, D. P. V., Guillou, S., Nguyen, K. D., \& Chauchat, J. (2012). Simulation of dredged sediment releases into homogeneous water using a two-phase model. Advances in Water Resources, 48, 102-112. doi $10.1016 / \mathrm{j}$.advwatres.2012.03.009.

Price, D., \& Laibe, G. (2015). Two-phase mixtures in SPH - A new approach. Proceedings of 10th international SPHERIC workshop, Parma, Italy, (pp. 68-75).

Ren, B., Li, C.-F., Yan, X., Lin, M. C., Bonet, J., \& Hu, S.-M. (2014). Multiple-fluid SPH Simulation Using a Mixture Model. ACM Transactions on Graphics, 33. doi 10.1145/2645703.

Richardson, J. F., \& Zaki, W. N. (1997). Sedimentation and fluidisation: Part I. Chemical Engineering Research and Design, 75, S82-S100. doi:10.1016/S0263-8762(97)80006-8

Rouse, H. (1937). Modern conceptions of the mechanics of turbulence. Transactions of the American Society of Civil Engineers, 102, 463-505.

Rusche, H. (2002). Computational fluid dynamics of dispersed two-phase flows at high phase fractions. Ph.D. thesis. 115-120. 
Shi, H., Yu, X., \& Dalrymple, R. A. (2017). Development of a two-phase SPH model for sediment laden flows. Computer Physics Communications, 221, 259-272. doi:10.1016/j.cpc.2017.08. 024 .

Vila, J. P. (1999). On particle weighted methods and Smoothed Particle Hydrodynamics. Mathematical Models and Methods in Applied Sciences, 09, 161-209. doi 10.1142/S0218202599000117.

Violeau, D. (2009). Dissipative forces for Lagrangian models in computational fluid dynamics and application to smoothed-particle hydrodynamics. Physical Review E, 80 . doi 10.1103/PhysRevE. 80.036705

Violeau, D. (2012). Fluid mechanics and the SPH method: theory and applications. chapter 5. Oxford University Press. doi 10.1093/acprof:oso/9780199655526.001.0001.

Violeau, D., \& Leroy, A. (2014). On the maximum time step in weakly compressible SPH. Journal of Computational Physics, 256, 388-415. doi:10.1016/j.jcp.2013.09.001

Wan, H., Liu, R., Pu, X., Zhang, H., \& Feng, J. (2018). Numerical simulation for the air entrainment of aerated flow with an improved multiphase SPH model. International Journal of Computational Fluid Dynamics, 31, 435-449. doi 10.1080/10618562.2017.1420175.

Wendland, H. (1995). Piecewise polynomial, positive definite and compactly supported radial functions of minimal degree. Advances in Computational Mathematics, 4, 389-396. doi:10. $1007 / \mathrm{BF} 02123482$ 